\title{
Random walks in Weyl chambers and crystals
}

\author{
Cédric Lecouvey, Emmanuel Lesigne and Marc Peigné
}

December 14, 2010

\begin{abstract}
We use Kashiwara crystal basis theory to associate a random walk $\mathcal{W}$ to each irreducible representation $V$ of a simple Lie algebra. This is achieved by endowing the crystal attached to $V$ with a (possibly non uniform) probability distribution compatible with its weight graduation. We then prove that the generalized Pitmann transform defined in 1 for similar random walks with uniform distributions yields yet a Markov chain. When the representation is minuscule, and the associated random walk has a drift in the Weyl chamber, we establish that this Markov chain has the same law as $\mathcal{W}$ conditionned to never exit the cone of dominant weights. For the defining representation $V$ of $\mathfrak{g l}_{\mathfrak{n}}$, we notably recover the main result of [19]. At the heart of our proof is a quotient version of a renewal theorem that we state in the context of general random walks in a lattice. This theorem also have applications in representation theory since it permits to precise the behavior of some outer multiplicities for large dominant weights.
\end{abstract}

Laboratoire de Mathématiques et Physique Théorique (UMR CNRS 6083)

Université François-Rabelais, Tours

Fédération de Recherche Denis Poisson - CNRS

Parc de Grandmont, 37200 Tours, France.

cedric.lecouvey@lmpt.univ-tours.fr

emmanuel.lesigne@lmpt.univ-tours.fr

marc.peigne@lmpt.univ-tours.fr 


\section{Contents}

1 Introduction

2 Background on representation theory 4

2.1 Root systems . . . . . . . . . . . . . . . . . . . 4

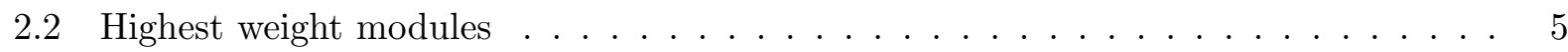

2.3 Minuscule representations . . . . . . . . . . . . . . . . . . . 7

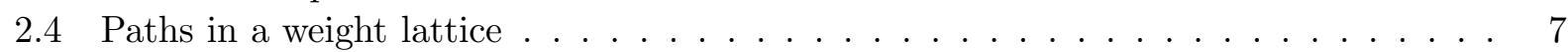

3 Background on Markov chains 8

3.1 Markov chains and conditioning . . . . . . . . . . . . . 8

3.2 Doob $h$-transforms . . . . . . . . . . . . . . . . . . . . . 9

3.3 Green function and Martin kernel . . . . . . . . . . . . . . . . . . 9

4 Quotient renewal theorem for a random walk in a cone $\quad \mathbf{1 0}$

4.1 Probability to stay in a cone . . . . . . . . . . . . . . . . . . 10

4.2 Local Limit Theorem . . . . . . . . . . . . . . . . . . . . . . . . 11

4.3 A quotient LLT for the random walk restricted to a cone . . . . . . . . . . . . 12

4.4 Renewal theorem . . . . . . . . . . . . . . . . . . . . . 14

4.5 A quotient renewal theorem for the random walk restricted to a cone . . . . . . . 15

5 Crystals and random walks $\quad \mathbf{1 7}$

5.1 Brief review on crystals . . . . . . . . . . . . . . . 17

5.2 Paths in weight lattices and crystals . . . . . . . . . . . . . . 20

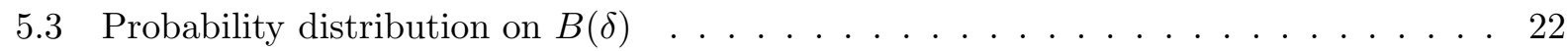

5.4 The random walk $\mathcal{W}$ in the weight lattice . . . . . . . . . . . 24

6 Markov chains in the Weyl chamber 26

6.1 The Markov chain $\mathcal{H} \ldots \ldots \ldots \ldots \ldots \ldots \ldots$

6.2 Intertwining operators . . . . . . . . . . . . . . . . . 27

7 Restriction to the Weyl chamber $\quad 28$

7.1 Doob transform of the random walk $\mathcal{W}$ restricted to the Weyl chamber . . . . . . . 28

7.2 Limit of $\psi$ along a drift . . . . . . . . . . . . . . . . . . . . . 29

7.3 Random walks $\mathcal{W}$ with fixed drift . . . . . . . . . . . . . . . . . 30

7.4 Transition matrix of $\mathcal{W}$ conditioned to never exit the Weyl chamber . . . . . . . . 31

8 Complementary results

8.1 Asymptotic behavior of the coefficients $f_{\lambda / \mu, \delta}^{\ell} \ldots \ldots \ldots \ldots \ldots \ldots$

8.2 Random walks defined from non irreducible representations . . . . . . . . . . 35

9 Appendix: Miscellaneous proofs $\quad 36$

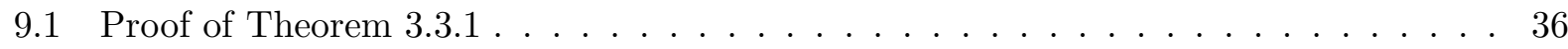

9.2 Proof of Lemma $4.3 .2 \ldots \ldots \ldots \ldots \ldots \ldots \ldots$ 


\section{Introduction}

The purpose of the article is to study some interactions between representation theory of simple Lie algebras over $\mathbb{C}$ and certain random walks defined on lattices in Euclidean spaces. We provide both results on random walks conditionned to never exit a cone and identities related to asymptotic representation theory.

The well-known ballot walk in $\mathbb{R}^{n}$ appears as a particular case of the random walks we consider here. Let $B=\left(\varepsilon_{1}, \ldots, \varepsilon_{n}\right)$ be the standard basis of $\mathbb{R}^{n}$. The ballot walk can be defined as the Markov chain $\left(\mathcal{W}_{\ell}=X_{1}+\cdots+X_{\ell}\right)_{\ell \geq 1}$ where $\left(X_{k}\right)_{k \geq 1}$ is a sequence of independent and identically distributed random variables taking values in the base $B$. Our main motivation is to generalize results due to O'Connell [19], 20] given the law of the random walk $\mathcal{W}=\left(\mathcal{W}_{\ell}\right)_{\ell \geq 1}$ conditioned to never exit the Weyl chamber $\bar{C}=\left\{x=\left(x_{1}, \ldots, x_{n}\right) \in \mathbb{R}^{n} \mid x_{1} \geq \cdots \geq x_{n}\right\}$. This is achieved in [19] by considering first a natural transformation $\mathfrak{P}$ which associates to any path with steps in $B$ a path in the Weyl chamber $\bar{C}$, next by checking that the image of the random walk $\mathcal{W}_{\ell}$ by this transformation is a Markov chain and finally by establishing that this Markov chain has the same law as $\mathcal{W}$ conditioned to never exit $\bar{C}$. The transformation $\mathfrak{P}$ is based on the RobinsonSchensted correspondence which maps the words on the ordered alphabet $\mathcal{A}_{n}=\{1<\cdots<n\}$ (regarded as finite paths in $\mathbb{R}^{n}$ ) on pairs of semistandard tableaux. It can be reinterpreted in terms of Kashiwara's crystal basis theory [13] (or equivalently in terms of the Littelmann path model). Each path of length $\ell$ with steps in $B$ is then interpreted as a vertex in the crystal $B\left(\omega_{1}\right)^{\otimes \ell}$ (see $\$ 5.1$ for basics on crystals) corresponding to the $\ell$-tensor power of the defining representation of $\mathfrak{s l}_{n}$. The transformation $\mathfrak{P}$ associates to each vertex $b \in B\left(\omega_{1}\right)^{\otimes \ell}$ the highest weight vertex of $B(b)$, where $B(b)$ denotes the connected component of $B\left(\omega_{1}\right)^{\otimes \ell}$ containing $b$.

Let $\mathfrak{g}$ be a simple Lie algebra over $\mathbb{C}$ with weight lattice $P \subset \mathbb{R}^{N}$ and dominant weights $P_{+}$ (see Section 2 for some background on root systems and representation theory). Write also $\bar{C}$ for the corresponding Weyl chamber. For any dominant weight $\delta \in P_{+}$, let $V(\delta)$ be the (finitedimensional) irreducible representation of $\mathfrak{g}$ of highest weight $\delta$ and denote by $B(\delta)$ its Kashiwara crystal graph. One can then consider the transformation $\mathfrak{P}$ which maps any $b \in B(\delta)^{\otimes \ell}$ on the highest weight vertex $\mathfrak{P}(b)$ of the connected component $B(b)$ of the graph $B(\delta)^{\otimes \ell}$ containing the vertex $b$. This simply means that $\mathfrak{P}(b)$ is the source vertex of $B(b)$ considered as an oriented graph. This transformation was introduced in [1]. As described in [1, it can be interpreted as a generalization of the Pitman transform for one-dimensional paths.

In order to define a random walk from the tensor powers $B(\delta)^{\otimes \ell}$, we need first to endow $B(\delta)$ with a probability distribution $\left(p_{a}\right)_{a \in B(\delta)}$. Contrary to [1] where the random walks considered are discrete version of Brownian motions, we will consider non uniform distributions on $B(\delta)$ and use random walks with drift in $C$. Once we have defined a probability on $B(\delta)$, it suffices to consider the product probability on $B(\delta)^{\otimes \ell}$ : the probability associated to the vertex $b=a_{1} \otimes \cdots \otimes a_{\ell} \in B(\delta)^{\otimes \ell}$ is then $p_{b}=p_{a_{1}} \cdots p_{a_{\ell}}$. In order to use Kashiwara crystal basis theory, it is also natural to impose that the distributions we consider on the crystal $B(\delta)^{\otimes \ell}$ are compatible with their weight graduation wt, that is $p_{b}=p_{b^{\prime}}$ whenever $\operatorname{wt}(b)=\operatorname{wt}\left(b^{\prime}\right)$. Since $\operatorname{wt}(b)=\operatorname{wt}\left(a_{1}\right)+\cdots+\operatorname{wt}\left(a_{\ell}\right)$, the two conditions $p_{b}=p_{a_{1}} \cdots p_{a_{\ell}}$ and $p_{b}=p_{b^{\prime}}$ whenever $\operatorname{wt}(b)=\operatorname{wt}\left(b^{\prime}\right)$ essentially impose that our initial distribution on $B(\delta)$ should be exponential with respect to the weight graduation. To be

more precise, recall that $B(\delta)$ is an oriented and colored graph with arrows $a \stackrel{i}{\rightarrow} a^{\prime}$ labelled by the simple roots $\alpha_{i}, i=1, \ldots, n$, of $\mathfrak{g}$. Moreover, we have then $\operatorname{wt}\left(a^{\prime}\right)=\operatorname{wt}(a)-\alpha_{i}$. We thus associate to each simple root $\alpha_{i}$ a positive real number $t_{i}$ and consider only probability distributions on $B(\delta)$ such that $a \stackrel{i}{\rightarrow} a^{\prime}$ implies $p_{a^{\prime}}=p_{a} \times t_{i}$. These distributions and their corresponding product 
on $B(\delta)^{\otimes \ell}$ are then compatible with the weight graduation as required. The uniform distribution corresponds to the case when $t_{1}=\cdots=t_{n}=1$.

The tensor power $B(\delta)^{\otimes \mathbb{N}}$ (defined as the projective limit of the crystals $B(\delta)^{\otimes \ell}$ ) can then also be endowed with a probability distribution of product ype. We define the random variable $\mathcal{W}_{\ell}$ on $B(\delta)^{\otimes \mathbb{N}}$ by $\mathcal{W}_{\ell}(b)=\operatorname{wt}(b(\ell))$ where $b(\ell)=\otimes_{k=1}^{\ell} a_{k} \in B(\delta)^{\otimes \ell}$ for any $b=\otimes_{k=1}^{+\infty} a_{k} \in B(\delta)^{\otimes \mathbb{N}}$. This yields a random walk $\mathcal{W}=\left(\mathcal{W}_{\ell}\right)_{\ell \geq 1}$ on $P$ whose transition matrix $\Pi_{\mathcal{W}}$ can be easily computed (see 23). Our next step is to introduce the random process $\mathcal{H}=\left(\mathcal{H}_{\ell}\right)_{\ell \geq 1}$ on $P_{+}$such that $\mathcal{H}_{\ell}(b)=$ $\mathrm{wt}(\mathfrak{P}(b(\ell)))$ for any $b \in B(\delta)^{\otimes \mathbb{N}}$. We prove in Theorem6.1.2 that $\mathcal{H}$ is a Markov chain and compute its transition matrix $\Pi_{\mathcal{H}}$ in terms of the Weyl characters of the irreducible representations $V(\lambda), \lambda \in$ $P_{+}$.

Write $\Pi_{\mathcal{W}}^{\bar{C}}$ for the restriction of $\Pi_{\mathcal{W}}$ to the Weyl chamber $\bar{C}$. Proposition 7.1.1 states that $\Pi_{\mathcal{H}}$ can be regarded as a Doob $h$-transform of $\Pi_{\mathcal{W}}^{\bar{C}}$ if and only if $\delta$ is a minuscule weight. As in [19] and [20], we use a theorem of Doob (Theorem 3.3.1) to compare the law of $\mathcal{W}$ conditioned to stay in $\bar{C}$ with the law of $\mathcal{H}$. Nevertheless the proofs in [19] and [20] use, as a key argument, asymptotic behaviors of the outer multiplicities in $V(\mu) \otimes V(\delta)^{\otimes \ell}$ when $\delta$ is the defining representation of $\mathfrak{s l}_{n}$. These limits seem very difficult to obtain for any minuscule representation by purely algebraic means. To overcome this problem, we establish Theorems 4.3.1 and 4.5.1 which can be seen as quotient Local Limit Theorem and quotient Renewal Theorem for large deviations of random walks conditioned to stay in a cone. They hold in the general context of random walks in a lattice with drift $m$ in the interior $C$ of a fixed closed cone $\bar{C}$. This yields Theorem 7.4 .2 equating the law of $\mathcal{W}$ conditioned to never exit $\bar{C}$ with that of $\mathcal{H}$ provided $\delta$ is a minuscule representation and $m=\mathbb{E}\left(\mathcal{W}_{1}\right)$ belongs to $C$.

The quotient Local Limit Theorem has also some applications in representation theory since it provides the asymptotic behavior for the outer multiplicities in $V(\mu) \otimes V(\delta)^{\otimes \ell}$ when $\delta$ is a minuscule representation (Theorem 8.1.1).

The paper is organized as follows. Sections 2 and 3 are respectively devoted to basics on representation theory of Lie algebras and Markov chains. In Section 4, we state and prove the probability results (Theorems 4.3.1 and 4.5.1). Section 5 details the construction of the random walk $\mathcal{W}$. The transition matrix of the Markov chain $\mathcal{H}$ is computed in Section 6 . The main results of Section 7 are Theorem 7.4 .2 and Corollary 7.4.3. This corollary gives an explicit formula for the probability that $\mathcal{W}$ remains forever in $\bar{C}$. Finally in Section 8 , we study random walks defined from non irreducible representations and prove Theorem 8.1.1.

\section{Background on representation theory}

We recall in the following paragraphs some classical background on representation theory of simple Lie algebras that we shall need in the sequel. For a complete review, the reader is referred to [3] or [10].

\section{$2.1 \quad$ Root systems}

Let $\mathfrak{g}$ be a simple Lie algebra over $\mathbb{C}$ and $\mathfrak{g}=\mathfrak{g}_{+} \oplus \mathfrak{h} \oplus \mathfrak{g}_{-}$a triangular decomposition. We shall follow the notation and convention of [3]. According to the Cartan-Killing classification, $\mathfrak{g}$ is characterized (up to isomorphism) by its root system. This root system is realized in an Euclidean space $\mathbb{R}^{N}$ with standard basis $B=\left(\varepsilon_{1}, \ldots, \varepsilon_{N}\right)$. We denote by $\Delta_{+}=\left\{\alpha_{i} \mid i \in I\right\}$ the set of simple roots of $\mathfrak{g}$, by $R_{+}$the (finite) set of positive roots and let $n=\operatorname{card}(I)$ for the rank of $\mathfrak{g}$. The root lattice of $\mathfrak{g}$ 
is the integral lattice $Q=\bigoplus_{i=1}^{n} \mathbb{Z} \alpha_{i}$. Write $\omega_{i}, i=1, \ldots, n$ for the fundamental weights associated to $\mathfrak{g}$. The weight lattice associated to $\mathfrak{g}$ is the integral lattice $P=\bigoplus_{i=1}^{n} \mathbb{Z} \omega_{i}$. It can be regarded as an integral sublattice of $\mathfrak{h}_{\mathbb{R}}^{*}$. We have $\operatorname{dim}(P)=\operatorname{dim}(Q)=n$ and $Q \subset P$.

The cone of dominant weights for $\mathfrak{g}$ is obtained by considering the positive integral linear combinations of the fundamental weights, that is

$$
P_{+}=\bigoplus_{i=1}^{n} \mathbb{N} \omega_{i} .
$$

The corresponding Weyl chamber is the cone $C=\bigoplus_{i=1}^{n} \mathbb{R}_{>0} \omega_{i}$. We also introduce its closure $\bar{C}=\bigoplus_{i=1}^{n} \mathbb{R}_{\geq 0} \omega_{i}$. In type $A$, we shall use the weight lattice of $\mathfrak{g l}_{n}$ rather than that of $\mathfrak{s l}_{n}$ for simplicity. We also introduce the Weyl group $W$ of $\mathfrak{g}$ which is the group generated by the reflections $s_{i}$ through the hyperplanes perpendicular to the simple root $\alpha_{i}, i=1, \ldots, n$. Each $w \in W$ may be decomposed as a product of the $s_{i}, i=1, \ldots, n$. All the minimal length decompositions of $w$ have the same length $l(w)$. For any weight $\beta$, the orbit $W \cdot \beta$ of $\beta$ under the action of $W$ intersects $P_{+}$in a unique point. We define a partial order on $P$ by setting $\mu \leq \lambda$ if $\lambda-\mu$ belongs to $Q_{+}=\bigoplus_{i=1}^{n} \mathbb{N} \alpha_{i}$.

\section{Examples 2.1.1}

1. For $\mathfrak{g}=\mathfrak{g l}_{n}, P_{+}=\left\{\lambda=\left(\lambda_{1}, \ldots, \lambda_{n}\right) \in \mathbb{Z}^{n} \mid \lambda_{1} \geq \cdots \geq \lambda_{n} \geq 0\right\}$ and $W$ coincides with the permutation group on $\mathcal{A}_{n}=\{1, \ldots, n\}$. We have $N=n$. For any $\sigma \in W$ and any $v=\left(v_{1}, \ldots, v_{n}\right), \sigma \cdot v=\left(v_{\sigma(1)}, \ldots, v_{\sigma(n)}\right)$.

2. For $\mathfrak{g}=\mathfrak{s p}_{2 n}, P_{+}=\left\{\lambda=\left(\lambda_{1}, \ldots, \lambda_{n}\right) \in \mathbb{Z}^{n} \mid \lambda_{1} \geq \cdots \geq \lambda_{n} \geq 0\right\}$ and $W$ coincides with the group of signed permutations on $\mathcal{C}_{n}=\{\bar{n}, \ldots, \overline{1}, 1, \cdots, n\}$, i.e. the permutations $w$ of $\mathcal{B}_{n}$ such that $\overline{w(x)}=w(\bar{x})$ for any $x \in \mathcal{B}_{n}$ (here we write $\bar{x}$ for $-x$ so that $\overline{\bar{x}}=x$ ). We have $N=n$. For any $\sigma \in W$ and any $v=\left(v_{1}, \ldots, v_{n}\right), \sigma \cdot v=\left(\theta_{1} v_{|\sigma(1)|}, \ldots, \theta_{n} v_{|\sigma(1)|}\right)$ where $\theta_{i}=1$ if $\sigma(i)$ is positive and $\theta_{i}=-1$ otherwise.

3. For $\mathfrak{g}=\mathfrak{s o}_{2 n+1}, P_{+}=\left\{\lambda=\left(\lambda_{1}, \ldots, \lambda_{n}\right) \in \mathbb{Z}^{n} \sqcup\left(\mathbb{Z}+\frac{1}{2}\right)^{n} \mid \lambda_{1} \geq \cdots \geq \lambda_{n} \geq 0\right\}$. The Weyl group $W$ is the same as for $\mathfrak{s p}_{2 n}$.

4. For $\mathfrak{g}=\mathfrak{s o}_{2 n}, P_{+}=\left\{\lambda=\left(\lambda_{1}, \ldots, \lambda_{n}\right) \in \mathbb{Z}^{n} \sqcup\left(\mathbb{Z}+\frac{1}{2}\right)^{n}\left|\lambda_{1} \geq \cdots \geq \lambda_{n-1} \geq\right| \lambda_{n} \mid \geq 0\right\}$. The Weyl group is the subgroup of the signed permutations on $\mathcal{B}_{n}$ switching and even number of signs.

\subsection{Highest weight modules}

Let $U(\mathfrak{g})$ be the enveloping algebra associated to $\mathfrak{g}$. Each finite dimensional $\mathfrak{g}$ (or $U(\mathfrak{g})$ )-module $M$ admits a decomposition in weight spaces

$$
M=\bigoplus_{\mu \in P} M_{\mu}
$$

where

$$
M_{\mu}:=\{v \in M \mid h(v)=\mu(h) v \text { for any } h \in \mathfrak{h}\}
$$


and $P$ is identified with a sublattice of $\mathfrak{h}_{\mathbb{R}}^{*}$. In particular, $\left(M \oplus M^{\prime}\right)_{\mu}=M_{\mu} \oplus M_{\mu}^{\prime}$. The Weyl group $W$ acts on the weights of $M$ and for any $\sigma \in W$, we have $\operatorname{dim} M_{\mu}=\operatorname{dim} M_{\sigma \cdot \mu}$. The character of $M$ is the Laurent polynomial in the variables $x_{1}, \ldots, x_{N}$

$$
\operatorname{char}(M)(x):=\sum_{\mu \in P} \operatorname{dim}\left(M_{\mu}\right) x^{\mu}
$$

where $\operatorname{dim}\left(M_{\mu}\right)$ is the dimension of the weight space $M_{\mu}$ and $x^{\mu}=x_{1}^{\mu_{1}} \cdots x_{N}^{\mu_{N}}$ with $\left(\mu_{1}, \ldots, \mu_{N}\right)$ the coordinates of $\mu$ on the standard basis $\left\{\varepsilon_{1}, \cdots, \varepsilon_{N}\right\}$.

The irreducible finite dimensional representations of $\mathfrak{g}$ are labelled by the dominant weights. For each dominant weight $\lambda \in P_{+}$, let $V(\lambda)$ be the irreducible representation of $\mathfrak{g}$ associated to $\lambda$. The category $\mathcal{C}$ of finite dimensional representations of $\mathfrak{g}$ over $\mathbb{C}$ is semisimple: each module decomposes into irreducible components. The category $\mathcal{C}$ is equivariant to the (semisimple) category of finite dimensional $U(\mathfrak{g})$-modules (over $\mathbb{C}$ ). Any finite dimensional $U(\mathfrak{g})$-module $M$ decomposes as a direct sum of irreducible

$$
M=\bigoplus_{\lambda \in P_{+}} V(\lambda)^{\oplus m_{M, \lambda}}
$$

where $m_{M, \lambda}$ is the multiplicity of $V(\lambda)$ in $M$. Here we slightly abuse the notation by also denoting by $V(\lambda)$ the irreducible f.d. $U(\mathfrak{g})$-module associated to $\lambda$.

When $M=V(\lambda)$ is irreducible, we set

$$
s_{\lambda}(x):=\operatorname{char}(M)(x)=\sum_{\mu \in P} K_{\lambda, \mu} x^{\mu}
$$

with $\operatorname{dim}\left(M_{\mu}\right)=K_{\lambda, \mu}$. Then $K_{\lambda, \mu} \neq 0$ only if $\mu \leq \lambda$. The characters can be computed from the Weyl character formula

$$
s_{\lambda}(x)=\frac{\sum_{w \in W} \varepsilon(w) x^{w(\lambda+\rho)-\rho}}{\prod_{\alpha \in R_{+}}\left(1-x^{-\alpha}\right)} .
$$

where $\varepsilon(w)=(-1)^{\ell(w)}$ and $\rho=\frac{1}{2} \sum_{\alpha \in R_{+}} \alpha_{i}$ is the half sum of positive roots.

Given $\delta, \mu$ in $P_{+}$and a nonnegative integer $\ell$, we define the tensor multiplicities $f_{\lambda / \mu, \delta}^{\ell}$ by

$$
V(\mu) \otimes V(\delta)^{\otimes \ell} \simeq \bigoplus_{\lambda \in P_{+}} V(\lambda)^{\oplus f_{\lambda / \mu, \delta}^{\ell}}
$$

For $\mu=0$, we set $f_{\lambda, \delta}^{\ell}=f_{\lambda / 0, \delta}^{\ell}$. When there is no risk of confusion, we write simply $f_{\lambda / \mu}^{\ell}$ (resp. $f_{\lambda}^{\ell}$ ) instead of $f_{\lambda / \mu, \delta}^{\ell}\left(\right.$ resp. $\left.f_{\lambda, \delta}^{\ell}\right)$. We also define the multiplicities $m_{\mu, \delta}^{\lambda}$ by

$$
V(\mu) \otimes V(\delta) \simeq \bigoplus_{\mu \rightsquigarrow \lambda} V(\lambda)^{\oplus m_{\mu, \delta}^{\lambda}}
$$

where the notation $\mu \rightsquigarrow \lambda$ means that $\lambda \in P_{+}$and $V(\lambda)$ appears as an irreducible component of $V(\mu) \otimes V(\delta)$. We have in particular $m_{\mu, \delta}^{\lambda}=f_{\lambda / \mu, \delta}^{1}$.

Lemma 2.2.1 Consider $\mu \in P_{+}$. Then for any $\beta \in P$, we have the relation

$$
\sum_{\lambda \in P_{+}, \mu \rightsquigarrow \lambda} m_{\mu, \delta}^{\lambda} K_{\lambda, \beta}=\sum_{\gamma \in P} K_{\mu, \gamma} K_{\delta, \beta-\gamma} .
$$


Proof. According to (3), one gets

$$
s_{\mu}(x) \times s_{\delta}(x)=\sum_{\mu \rightsquigarrow \lambda} \sum_{\beta \in P} m_{\mu, \delta}^{\lambda} K_{\lambda, \beta} x^{\beta} .
$$

On the other hand

$$
s_{\mu}(x) \times s_{\delta}(x)=\sum_{\gamma \in P} K_{\mu, \gamma} x^{\gamma} \sum_{\xi \in P} K_{\delta, \xi} x^{\xi}=\sum_{\beta \in P} \sum_{\gamma \in P} K_{\mu, \gamma} K_{\delta, \beta-\gamma} x^{\beta} .
$$

By comparing both expressions, we derive the expected relation.

\subsection{Minuscule representations}

The irreducible representation $V(\delta)$ is said minuscule when the orbit $W \cdot \delta$ of the highest weight $\delta$ under the action of the Weyl group $W$ contains all the weights of $V(\delta)$. In that case, the dominant weight $\delta$ is also called minuscule. The minuscule weights are fundamental weights and each weight space in $V(\delta)$ has dimension 1 . They are given by the following table

\begin{tabular}{|l|l|l|l|}
\hline type & minuscule weights & $N$ & decomposition on $B$ \\
\hline$A_{n}$ & $\omega_{i}, i=1, \ldots, n$ & $n+1$ & $\omega_{i}=\varepsilon_{1}+\cdots+\varepsilon_{i}$ \\
\hline$B_{n}$ & $\omega_{n}$ & $n$ & $\omega_{n}=\frac{1}{2}\left(\varepsilon_{1}+\cdots+\varepsilon_{n}\right)$ \\
\hline$C_{n}$ & $\omega_{1}$ & $n$ & $\omega_{1}=\varepsilon_{1}$ \\
\hline$D_{n}$ & $\omega_{1}, \omega_{n-1}, \omega_{n}$ & $n$ & $\omega_{1}=\varepsilon_{1}, \omega_{n+t}=\frac{1}{2}\left(\varepsilon_{1}+\cdots+\varepsilon_{n}\right)+t \varepsilon_{n}, t \in\{-1,0\}$ \\
\hline$E_{6}$ & $\omega_{1}, \omega_{6}$ & 8 & $\omega_{1}=\frac{2}{3}\left(\varepsilon_{8}-\varepsilon_{7}-\varepsilon_{6}\right), \omega_{6}=\frac{1}{3}\left(\varepsilon_{8}-\varepsilon_{7}-\varepsilon_{6}\right)+\varepsilon_{5}$ \\
\hline$E_{7}$ & $\omega_{7}$ & 8 & $\omega_{7}=\varepsilon_{6}+\frac{1}{2}\left(\varepsilon_{8}-\varepsilon_{7}\right)$. \\
\hline
\end{tabular}

Here, the four infinite families $A_{n}, B_{n}, C_{n}$ and $D_{n}$ correspond respectively to the classical Lie algebras $\mathfrak{g}=\mathfrak{g l}_{n}, \mathfrak{g}=\mathfrak{s o}_{2 n+1}, \mathfrak{g}=\mathfrak{s p}_{2 n}$ and $\mathfrak{g}=\mathfrak{s o}_{2 n}$. When $\delta$ is minuscule, one may check that each nonzero multiplicity $m_{\mu, \delta}^{\lambda}$ in (3) is equal to 1 .

Remark: We will also need the following classical properties of minuscule representations.

1. If $\delta$ is minuscule, then for any $\lambda, \mu \in P_{+}$, one gets $K_{\delta, \lambda-\mu}=m_{\mu, \delta}^{\lambda} \in\{0,1\}$.

2. If $\delta$ is not a minuscule weight, there exists a dominant weight $\kappa \neq \delta$ such that $\kappa$ is a weight for $V(\delta)$, that is $K_{\delta, \kappa} \neq 0$. This follows from the fact that $V(\delta)$ contains a weight $\beta$ which does not belong to $W \cdot \delta$. Then we can take $\kappa=P_{+} \cap W \cdot \beta$.

\subsection{Paths in a weight lattice}

Consider $\delta$ a dominant weight associated to the Lie algebra $\mathfrak{g}$. We denote by $Z_{\gamma}(\delta, \ell)$ the set of paths of length $\ell$ in the weight lattice $P$ starting at $\gamma \in P$ with steps the set of weights of $V(\delta)$, that is the weights $\beta$ such that $V(\delta)_{\beta} \neq\{0\}$. When $\gamma=0$, we write for short $Z(\delta, \ell)=Z_{0}(\delta, \ell)$. A path in $Z_{\gamma}(\delta, \ell)$ can be identified with a sequence $\left(\mu^{(0)}, \mu^{(1)} \ldots, \mu^{(\ell)}\right)$ of weights of $V(\delta)$ such that $\mu^{(0)}=\gamma$. The position at the $k$-th step corresponds to the weight $\mu^{(k)}$. When $\delta$ is a minuscule weight, the paths of $Z_{\gamma}(\delta, \ell)$ will be called minuscule. We will see in $\S 5.1$ that the paths in $Z(\delta, \ell)$ can then be identified with the vertices of the $\ell$-th tensor product of the Kashiwara crystal graph associated to the representation $V(\delta)$.

Assume $\gamma \in P_{+}$. We denote by $Z_{\gamma}^{+}(\delta, \ell)$ the set of paths of length $\ell$ which never exit the closed Weyl chamber $\bar{C}$. Since $\bar{C}$ is convex, this is equivalent to say that $\mu^{(k)} \in P_{+}$for any $k=0, \ldots, \ell$. 


\section{Examples 2.4.1}

1. For $\mathfrak{g}=\mathfrak{g l}_{n}$ and $\delta=\omega_{1}$, the representation $V\left(\omega_{1}\right)$ has dimension $n$. The weights of $V\left(\omega_{1}\right)$ are the vectors $\varepsilon_{1}, \ldots, \varepsilon_{n}$ of the standard basis $B$. The paths $Z\left(\omega_{1}, \ell\right)$ are those appearing in the classical ballot problem.

2. For $\mathfrak{g}=\mathfrak{s p}_{2 n}$ and $\delta=\omega_{1}$, the representation $V\left(\omega_{1}\right)$ has dimension $2 n$. The weights of $V\left(\omega_{1}\right)$ are the vectors $\pm \varepsilon_{1}, \ldots, \pm \varepsilon_{n}$. In particular a path of $Z_{\gamma}\left(\omega_{1}, \ell\right)$ can return to its starting point $\gamma$.

3. For $\mathfrak{g}=\mathfrak{s o}_{2 n+1}$ and $\delta=\omega_{1}$, the representation $V\left(\omega_{1}\right)$ has dimension $2 n+1$. The weights of $V\left(\omega_{1}\right)$ are 0 and the vectors $\pm \varepsilon_{1}, \ldots, \pm \varepsilon_{n}$. The representation $V\left(\omega_{1}\right)$ is not minuscule.

4. For $\mathfrak{g}=\mathfrak{s o}_{2 n+1}$ and $\delta=\omega_{n}$, the representation $V(\delta)$ has dimension $2^{n}$. The coordinates of the weights appearing in $V(\delta)$ on the standard basis are of the form $\left(\beta_{1}, \ldots, \beta_{n}\right)$ where for any $i=1, \ldots, n, \beta_{i}= \pm \frac{1}{2}$.

Remark: In type $A$, the coordinates on the standard basis of the weights appearing in any representation are always nonnegative. This notably implies that a path in $Z_{\gamma}(\delta, \ell)$ can attain a fixed point of $\mathbb{R}^{N}$ at most one time. This special property does not hold in general for the paths of $Z_{\gamma}(\delta, \ell)$ in types other than type $A$. Indeed, the sign of the weight coordinates can be modified under the action of the Weyl group. In particular $Z_{\gamma}(\delta, \ell)$ contains paths of length $\ell>0$ which return to $\gamma$. This phenomenon introduces some complications in the probabilistic estimations which follows.

\section{Background on Markov chains}

\subsection{Markov chains and conditioning}

Consider a probability space $(\Omega, \mathcal{T}, \mathbb{P})$ and a countable set $M$. Let $Y=\left(Y_{\ell}\right)_{\ell \geq 1}$ be a sequence of random variables defined on $\Omega$ with values in $M$. The sequence $Y$ is a Markov chain when

$$
\mathbb{P}\left(Y_{\ell+1}=y_{\ell+1} \mid Y_{\ell}=y_{\ell}, \ldots, Y_{1}=y_{1}\right)=\mathbb{P}\left(Y_{\ell+1}=y_{\ell+1} \mid Y_{\ell}=y_{\ell}\right)
$$

for any any $\ell \geq 1$ and any $y_{1}, \ldots, y_{\ell}, y_{\ell+1} \in M$. The Markov chains considered in the sequel will also be assumed time homogeneous, that is $\mathbb{P}\left(Y_{\ell+1}=y_{\ell+1} \mid Y_{\ell}=y_{\ell}\right)=\mathbb{P}\left(Y_{\ell}=y_{\ell+1} \mid Y_{\ell-1}=y_{\ell}\right)$ for any $\ell \geq 2$. For all $x, y$ in $M$, the transition probability from $x$ to $y$ is then defined by

$$
\Pi(x, y)=\mathbb{P}\left(Y_{\ell+1}=y \mid Y_{\ell}=x\right)
$$

and we refer to $\Pi$ as the transition matrix of the Markov chain $Y$. The distribution of $Y_{1}$ is called the initial distribution of the chain $Y$. It is well known that the initial distribution and the transition probability determine the law of the Markov chain and that given a probability distribution and a transition matrix on $\mathrm{M}$, there exists an associated Markov chain.

An example of Markov chain is given by random walk on a group. Suppose that $M$ has a group structure and that $\nu$ is a probability measure on $M$; the random walk of law $\nu$ is the Markov chain with transition probabilities $\Pi(x, y)=\nu\left(x^{-1} y\right)$; this Markov chain starting at the neutral element of $M$ can be realized has $\left(X_{1} X_{2} \ldots X_{\ell}\right)_{\ell \geq 1}$ where $\left(X_{\ell}\right)_{\ell \geq 1}$ is a sequence of independent and 
identically distributed random variables, with law $\nu$.

Let $Y$ be a Markov chain on $(\Omega, \mathcal{T}, \mathbb{P})$, whose initial distribution has full support, i.e. $\mathbb{P}\left(Y_{1}=\right.$ $x)>0$ for any $x \in M$. Let $\mathcal{C}$ be a nonempty subset of $M$ and consider the event $S=\left(Y_{\ell} \in \mathcal{C}\right.$ for any $\ell \geq 1)$. Assume that $\mathbb{P}\left(S \mid Y_{1}=\lambda\right)>0$ for all $\lambda \in \mathcal{C}$. This implies that $\mathbb{P}[S]>0$, and we can consider the conditional probability $\mathbb{Q}$ relative to this event: $\mathbb{Q}[\cdot]=\mathbb{P}[\cdot \mid S]$.

It is easy to verify that, under this new probability $\mathbb{Q}$, the sequence $\left(Y_{\ell}\right)$ is still a Markov chain, with values in $\mathcal{C}$, and with transitions probabilities given by

$$
\mathbb{Q}\left[Y_{\ell+1}=\lambda \mid Y_{\ell}=\mu\right]=\mathbb{P}\left[Y_{\ell+1}=\lambda \mid Y_{\ell}=\mu\right] \frac{\mathbb{P}\left[S \mid Y_{1}=\lambda\right]}{\mathbb{P}\left[S \mid Y_{1}=\mu\right]}
$$

We will denote by $Y^{\mathcal{C}}$ this Markov chain and by $\Pi^{\mathcal{C}}$ the restriction of the transition matrix $\Pi$ to the entries which belong to $\mathcal{C}$ (in other words $\Pi^{\mathcal{C}}=(\Pi(\lambda, \mu))_{\lambda, \mu \in \mathcal{C}}$ ).

\subsection{Doob $h$-transforms}

A substochastic matrix on the countable set $M$ is a map $\Pi: M \times M \rightarrow[0,1]$ such that $\sum_{y \in M} \Pi(x, y) \leq$ 1 for any $x \in M$. If $\Pi, \Pi^{\prime}$ are substochastic matrices on $M$, we define their product $\Pi \times \Pi^{\prime}$ as the substochastic matrix given by the ordinary product of matrices:

$$
\Pi \times \Pi^{\prime}(x, y)=\sum_{z \in M} \Pi(x, z) \Pi^{\prime}(z, y) .
$$

The matrix $\Pi^{\mathcal{C}}$ defined in the previous subsection is an example of substochastic matrix.

A function $h: M \rightarrow \mathbb{R}$ is harmonic for the substochastic transition matrix $\Pi$ when we have $\sum_{y \in M} \Pi(x, y) h(y)=h(x)$ for any $x \in M$. Consider a strictly positive harmonic function $h$. We can then define the Doob transform of $\Pi$ by $h$ (also called the $h$-transform of $\Pi$ ) setting

$$
\Pi_{h}(x, y)=\frac{h(y)}{h(x)} \Pi(x, y)
$$

We then have $\sum_{y \in M} \Pi_{h}(x, y)=1$ for any $x \in M$. Thus $\Pi_{h}$ can be interpreted as the transition matrix for a certain Markov chain.

An example is given in the second part of the previous subsection (see formula (4)): the state space is now $\mathcal{C}$, the substochastic matrix is $\Pi^{\mathcal{C}}$ and the harmonic function is $h_{\mathcal{C}}(\lambda):=\mathbb{P}\left[S \mid Y_{1}=\lambda\right]$; the transition matrix $\Pi_{h_{\mathcal{C}}}^{\mathcal{C}}$ is the transition matrix of the Markov chain $Y^{\mathcal{C}}$.

\subsection{Green function and Martin kernel}

Let $\Pi$ be a substochastic matrix on the set $M$. Its Green function is defined as the series

$$
\Gamma(x, y)=\sum_{\ell \geq 0} \Pi^{\ell}(x, y) .
$$

(If $\Pi$ is the transition matrix of a Markov chain, $\Gamma(x, y)$ is the expected value of the number of passage at $y$ of the Markov chain starting at $x$.) 
Assume there exists $x^{*}$ in $M$ such that $0<\Gamma\left(x^{*}, y\right)<\infty$ for any $y \in M$. Fix such a point $x^{*}$. The Martin kernel associated to $\Pi$ (with reference point $x^{*}$ ) is then defined by

$$
K(x, y)=\frac{\Gamma(x, y)}{\Gamma\left(x^{*}, y\right)} .
$$

Consider a positive harmonic function $h$ and denote by $\Pi_{h}$ the $h$-transform of $\Pi$. Consider the Markov chain $Y^{h}=\left(Y_{\ell}^{h}\right)_{\ell \geq 1}$ starting at $x^{*}$ and whose transition matrix is $\Pi_{h}$.

Theorem 3.3.1 (Doob) Assume that there exists a function $f: M \rightarrow \mathbb{R}$ such that for all $x \in M$, $\lim _{\ell \rightarrow+\infty} K\left(x, Y_{\ell}^{h}\right)=f(x)$ almost surely. Then there exists a positive real constant $c$ such that $f=c h$.

For the sake of completeness, we detail a proof of this Theorem in the Appendix.

\section{Quotient renewal theorem for a random walk in a cone}

The purpose of this section is to establish a renewal theorem for a random walk forced to stay in a cone. We state this theorem in the weak form of a quotient theorem : see Theorem 4.5.1, This result is a key ingredient in our proof of Theorem 7.4 .2 ; it is a purely probabilistic result whose proof can be read independently of the reminder of the article.

We begin by the statement and proof of a quotient local limit theorem for a random walk forced to stay in a cone (Theorem 4.3.1). This local limit theorem is easier to establish than the renewal one and the ideas of its proof will be reinvested in the proof of Theorem 4.5.1.

\subsection{Probability to stay in a cone}

Let $\left(X_{\ell}\right)_{\ell \geq 1}$ be a sequence of random variables in an Euclidean space $\mathbb{R}^{n}$, independent and identically distributed, defined on a probability space $(\Omega, \mathcal{T}, \mathbb{P})$. We assume that these variables have moment of order 1 and denote by $m$ their common mean. Let us denote by $\left(S_{\ell}\right)_{\ell \geq 0}$ the associated random walk defined by $S_{0}=0$ and $S_{\ell}:=X_{1}+\cdots+X_{\ell}$. We consider a cone $\mathcal{C}$ in $\mathbb{R}^{n}$. We assume it contains an open convex sub-cone $\mathcal{C}_{0}$ such that $m \in \mathcal{C}_{0}$ and $\mathbb{P}\left[X_{\ell} \in \mathcal{C}_{0}\right]>0$. First, one gets the

\section{Lemma 4.1.1}

$$
\mathbb{P}\left[\forall \ell \geq 1, S_{\ell} \in \mathcal{C}\right]>0 .
$$

Proof. It suffices to prove the lemma for $\mathcal{C}_{0}$, that is, we can assume (and we will in the sequel) that $\mathcal{C}$ is open and convex. Fix $a$ in $\mathcal{C}$ such that $\mathbb{P}\left[X_{\ell} \in B(a, \varepsilon)\right]>0$, for any $\varepsilon>0$. Such an element does exist since the cone is charged by the law of $X$.

By the strong law of large numbers, the sequence $\left(\frac{1}{\ell} S_{\ell}\right)$ converges almost surely to $m$. Therefore, almost surely, one gets $S_{\ell} \in \mathcal{C}$ for any large enough $\ell$, that is

$$
\mathbb{P}\left[\exists L, \forall \ell \geq L, S_{\ell} \in \mathcal{C}\right]=\lim _{L \rightarrow+\infty} \mathbb{P}\left[\forall \ell \geq L, S_{\ell} \in \mathcal{C}\right]=1 .
$$

For any $x \in \mathbb{R}^{n}$, there exists $k \in \mathbb{N}$ such that $x+k a \in \mathcal{C}$. Thus

$$
\left(\forall \ell \geq L, S_{\ell} \in \mathcal{C}\right)=\bigcup_{k \geq 0}\left(\left(\forall \ell \geq L, S_{\ell} \in \mathcal{C}\right) \text { and }\left(\forall \ell<L, S_{\ell}+k a \in \mathcal{C}\right)\right)
$$


and therefore $\left(\forall \ell \geq L, S_{\ell} \in \mathcal{C}\right) \subset \bigcup_{k \geq 0}\left(\forall \ell, S_{\ell}+k a \in \mathcal{C}\right)$ since the cone is stable under addition. Hence, there exists $k \geq 0$ such that $\mathbb{P}\left[\forall \ell, S_{\ell}+k a \in \mathcal{C}\right]>0$. Fix such a $k$ and $\delta>0$ such that $B(a, \delta) \subset \mathcal{C}$. If $X_{\ell} \in B\left(a, \frac{\delta}{k+1}\right)$ for any $\ell \leq k+1$, then $S_{k+1}-k a \in B(a, \delta)$. We have

$$
\begin{aligned}
& \mathbb{P}\left[\forall \ell \geq 1, S_{\ell} \in \mathcal{C}\right] \geq \\
& \quad \mathbb{P}\left[\left(\left(\forall \ell \leq k+1, X_{\ell} \in B\left(a, \frac{\delta}{k+1}\right)\right) \text { and }\left(\forall \ell>k+1, S_{\ell}-S_{k+1}+k a \in \mathcal{C}\right)\right]=\right. \\
& \left(\mathbb{P}\left[X \in B\left(a, \frac{\delta}{k+1}\right)\right]\right)^{k+1} \mathbb{P}\left[\forall \ell, S_{\ell}+k a \in \mathcal{C}\right]>0 .
\end{aligned}
$$

\subsection{Local Limit Theorem}

We now assume that the support $S_{\mu}$ of the law $\mu$ of the random variables $X_{l}$ is a subset of $\mathbb{Z}^{n}$. We suppose that $\mu$ is adapted on $\mathbb{Z}^{n}$, which means that the group $\left\langle S_{\mu}\right\rangle$ generated by the elements of $S_{\mu}$ is equal to $\mathbb{Z}^{n}$.

The local limit theorem precises the behavior as $\ell \rightarrow+\infty$ of the probability $\mathbb{P}\left[S_{\ell}=g\right]$ for $g \in \mathbb{Z}^{n}$. It thus appears a phenomenon of "periodicity". For instance, for the classical random walk to the closest neighbor on $\mathbb{Z}$, the walk $\left(S_{\ell}\right)_{\ell \geq 1}$ may visit an odd site only at an odd time ; this is due to the fact that in this case the support of $\mu$ is included in $2 \mathbb{Z}+1$, that is a coset of a proper subgroup of $\mathbb{Z}$.

Here is a classical definition : we say that the law $\mu$ is aperiodic if there is no proper subgroup $G$ of $\left\langle S_{\mu}\right\rangle$ and vector $b \in\left\langle S_{\mu}\right\rangle$ such that $S_{\mu} \subset b+G$. One may easily check that if the law $\mu$ is aperiodic then translating $\mu$ by $-b$ with $b \in S_{\mu}$ leads to a new law $\mu^{\prime}$ which is aperiodic and such that $G=\left\langle S_{\mu^{\prime}}\right\rangle$ is a proper subgroup of $\left\langle S_{\mu}\right\rangle$.

Let us give some various examples :

Examples :

1. Denote by $\left(e_{i}\right)_{1 \leq i \leq n}$ the canonical basis of $\mathbb{R}^{n}$. If the variables $X_{\ell}$ take their values in the set $\left\{e_{i} \mid 1 \leq i \leq n\right\} \cup\{0\}$, if $\mathbb{P}\left[X_{\ell}=e_{i}\right]>0$ for any $i$ and if $\mathbb{P}\left[X_{\ell}=0\right]>0$, then $\left\langle S_{\mu}\right\rangle=\mathbb{Z}^{n}$ and the law is aperiodic.

2. If the variables $X_{\ell}$ take their values in $\left\{ \pm e_{i} \mid 1 \leq i \leq n\right\}$, if $\mathbb{P}\left[X_{\ell}=e_{i}\right]>0$ for any $i$ and if $\mathbb{P}\left[X_{\ell}=-e_{i}\right]>0$ for at least one $i$, then we can take $b=e_{1}$ and $G=\left\{\left(x_{1}, x_{2}, \ldots, x_{n}\right) \in \mathbb{Z}^{n} \mid\right.$ $x_{1}+x_{2}+\ldots+x_{n}$ is even $\}$ is the subgroup of $\mathbb{Z}^{n}$ generated by the $e_{i}+e_{j}, 1 \leq i, j \leq n$.

3. If the variables $X_{\ell}$ take their values in $\left\{e_{i} \mid 1 \leq i \leq n\right\}$ and if $\mathbb{P}\left[X_{\ell}=e_{i}\right]>0$ for any $i$, then we can take $b=e_{1}$ and $G$ is the $n$-1-dimensional subgroup generated by the vectors $e_{i}-e_{1}, 2 \leq i \leq n$.

The translation by $-b$ thus permits to limit ourselves to aperiodic random walks in a group of suitable dimension $d$, (with possibly $d<n$ ). By replacing the random walk $\left(S_{\ell}\right.$ ) by the random walk $\left(S_{\ell}-\ell b\right)$, we can therefore restrict ourselves to an adapted aperiodic random walk in the discrete group $G$.

We denote by $d$ the dimension of the group $G$ once this translation is performed. We also assume that the random walk admits a moment of order 2 . We write $m$ for the mean vector of $X$ 
and $\Gamma$ for the covariance matrix. A classical form of the local limit theorem is the following:

$$
\lim _{\ell \rightarrow+\infty} \sup _{g \in G}\left|\frac{\ell^{d / 2}}{v(G)} \mathbb{P}\left[S_{\ell}=g\right]-\mathcal{N}_{\Gamma}\left(\frac{1}{\sqrt{\ell}}(g-\ell m)\right)\right|=0
$$

where $\mathcal{N}_{\Gamma}$ is the Gaussian density

$$
\mathcal{N}_{\Gamma}(x)=(2 \pi)^{-d / 2}(\operatorname{det} \Gamma)^{-1 / 2} \exp \left(-\frac{1}{2} x \cdot \Gamma^{-1} \cdot{ }^{t} x\right), \quad x \in \mathbb{R}^{d},
$$

and $v(G)$ is the volume of an elementary cell of $G$. This result gives an equivalent of $\mathbb{P}\left[S_{\ell}=g_{\ell}\right]$ providing that $g_{\ell}-\ell m=O(\sqrt{\ell})$. The local limit theorem for large deviations (see the original article [22] or the classical book [9]) yields and equivalent when $g_{\ell}-\ell m=o(\ell)$. It takes a particularly simple form when $g_{\ell}-\ell m=o\left(\ell^{2 / 3}\right)$.

Theorem 4.2.1 Assume the random variables $X_{\ell}$ have an exponential moment, that is, there exists $t>0$ such that $\mathbb{E}\left[\exp \left(t\left|X_{\ell}\right|\right)\right]<+\infty$. Let $\left(a_{\ell}\right)$ be a sequence of real numbers such that $\lim a_{\ell} \ell^{-2 / 3}=0$. Then, when $\ell$ tends to infinity, we have

$$
\mathbb{P}\left[S_{\ell}=g\right] \sim v(G) \ell^{-d / 2} \mathcal{N}_{\Gamma}\left(\frac{1}{\sqrt{\ell}}(g-\ell m)\right)
$$

uniformly in $g \in G$ such that $\|g-\ell m\| \leq a_{\ell}$.

Under the hypotheses of Theorem 4.2.1, we derive the following equivalent. Given $\left(g_{\ell}\right),\left(h_{\ell}\right)$ two sequences in $G$ such that $\lim \ell^{-2 / 3}\left\|g_{\ell}-\ell m\right\|=0$ and $\lim \ell^{-1 / 2}\left\|h_{\ell}\right\|=0$, we have

$$
\mathbb{P}\left[S_{\ell}=g_{\ell}+h_{\ell}\right] \sim \mathbb{P}\left[S_{\ell}=g_{\ell}\right]
$$

Observe that there exists a stronger version of this result where the exponent $2 / 3$ is replaced by 1 but the equivalent obtained is more complicated, and we will not need it in the present article.

\subsection{A quotient LLT for the random walk restricted to a cone}

We assume that the hypotheses of the previous Subsection are satisfied.

Theorem 4.3.1 Assume the random variables $X_{\ell}$ are almost surely bounded. Let $\left(g_{\ell}\right),\left(h_{\ell}\right)$ be two sequences in $G$ and $\alpha<2 / 3$ such that $\lim \ell^{-\alpha}\left\|g_{\ell}-\ell m\right\|=0$ and $\lim \ell^{-1 / 2}\left\|h_{\ell}\right\|=0$. Then, when $\ell$ tends to infinity, we have

$$
\mathbb{P}\left[S_{1} \in \mathcal{C}, \ldots, S_{\ell} \in \mathcal{C}, S_{\ell}=g_{\ell}+h_{\ell}\right] \sim \mathbb{P}\left[S_{1} \in \mathcal{C}, \ldots, S_{\ell} \in \mathcal{C}, S_{\ell}=g_{n}\right]
$$

The following lemma will play a crucial role; in order to keep a direct way to our principal results, we postpone its proof in an appendix.

Lemma 4.3.2 Assume the random variables $X_{\ell}$ are almost surely bounded. Let $\left.\alpha \in\right] 1 / 2,2 / 3[$. If the sequence $\left(\ell^{-\alpha}\left\|g_{\ell}-\ell m\right\|\right)$ is bounded, then there exists $c>0$ such that, for all large enough $\ell$,

$$
\mathbb{P}\left[S_{1} \in \mathcal{C}, \ldots, S_{\ell} \in \mathcal{C}, S_{\ell}=g_{\ell}\right] \geq \exp \left(-c \ell^{\alpha}\right) .
$$


In the sequel, we will use the following notation : if $\left(u_{\ell}\right)$ and $\left(v_{\ell}\right)$ are two real sequences, we write $u_{\ell} \preceq v_{\ell}$ when there exists a constant $\kappa>0$ such that $u_{\ell} \leq \kappa v_{\ell}$ for all large enough $\ell$.

Proof of Theorem 4.3.1. Fix a real number $\beta$ such that $\frac{1}{2}<\alpha<\beta<\frac{2}{3}$ and set $b_{\ell}=\left[\ell^{\beta}\right]$. Let $\delta>0$ be such that $B(m, \delta) \subset \mathcal{C}$. Set $B_{\ell}=B(\ell m, \ell \delta)$.

For any $\ell \geq 1$ we have $B_{\ell} \subset \mathcal{C}$. We are going to establish that

$$
Q_{\ell}:=\frac{\mathbb{P}\left[S_{1} \in \mathcal{C}, \ldots, S_{\ell} \in \mathcal{C}, S_{\ell}=g_{\ell}\right]}{\mathbb{P}\left[S_{1} \in \mathcal{C}, \ldots, S_{b_{\ell}} \in \mathcal{C}, S_{b_{\ell}} \in B_{b_{\ell}}, S_{\ell}=g_{\ell}\right]} \rightarrow 1 .
$$

By the Cramer-Chernoff large deviations inequality, there exists $c=c(\delta)>0$ such that

$$
\mathbb{P}\left[S_{b_{\ell}} \notin B_{b_{\ell}}\right]=\mathbb{P}\left[\left\|S_{b_{\ell}}-b_{\ell} m\right\| \geq b_{\ell} \delta\right] \leq \exp \left(-c b_{\ell}\right) \preceq \exp \left(-c l^{\beta}\right) .
$$

By Lemma 4.3.2, there also exists $c^{\prime}>0$ such that

$$
\mathbb{P}\left[S_{1} \in \mathcal{C}, \ldots, S_{\ell} \in \mathcal{C}, S_{\ell}=g_{\ell}\right] \geq \exp \left(-c^{\prime} \ell^{\alpha}\right) .
$$

Since $\alpha<\beta$, we thus have

$$
\mathbb{P}\left[S_{b_{\ell}} \notin B_{b_{\ell}}\right]=o\left(\mathbb{P}\left[S_{1} \in \mathcal{C}, \ldots, S_{\ell} \in \mathcal{C}, S_{\ell}=g_{\ell}\right]\right) .
$$

This gives

$$
\mathbb{P}\left[S_{1} \in \mathcal{C}, \ldots, S_{\ell} \in \mathcal{C}, S_{b_{\ell}} \in B_{b_{\ell}}, S_{\ell}=g_{\ell}\right] \sim \mathbb{P}\left[S_{1} \in \mathcal{C}, \ldots, S_{\ell} \in \mathcal{C}, S_{\ell}=g_{\ell}\right]
$$

and in particular for any large enough $\ell$

$$
\mathbb{P}\left[S_{1} \in \mathcal{C}, \ldots, S_{b_{\ell}} \in \mathcal{C}, S_{b_{\ell}} \in B_{b_{\ell}}, S_{\ell}=g_{\ell}\right] \geq \frac{1}{2} \mathbb{P}\left[S_{1} \in \mathcal{C}, \ldots, S_{\ell} \in \mathcal{C}, S_{\ell}=g_{\ell}\right]
$$

Now set

$$
U_{\ell}:=\frac{\mathbb{P}\left[S_{1} \in \mathcal{C}, \ldots, S_{\ell} \in \mathcal{C}, S_{b_{\ell}} \in B_{b_{\ell}}, S_{\ell}=g_{\ell}\right]}{\mathbb{P}\left[S_{1} \in \mathcal{C}, \ldots, S_{b_{\ell}} \in \mathcal{C}, S_{b_{\ell}} \in B_{b_{\ell}}, S_{\ell}=g_{\ell}\right]} .
$$

Write $\varepsilon=\operatorname{dist}\left(m, \mathcal{C}^{c}\right)$, and recall that $\varepsilon>0$. So, when the walk goes out the cone, its distance to the point $k m$ is at least $k \varepsilon$.

We have,

$$
0 \leq 1-U_{\ell} \leq \frac{\sum_{k \geq 1} \mathbb{P}\left[S_{b_{\ell}+k} \notin \mathcal{C}\right]}{\mathbb{P}\left[S_{1} \in \mathcal{C}, \ldots, S_{b_{\ell}} \in \mathcal{C}, S_{b_{\ell}} \in B_{b_{\ell}}, S_{\ell}=g_{\ell}\right]} .
$$

Thus by (8) and our choice of $\varepsilon$, we have for any sufficiently large $\ell$,

$$
\left|1-U_{\ell}\right| \leq 2 \frac{\sum_{k \geq b_{\ell}} \mathbb{P}\left[\left\|S_{k}-k m\right\| \geq k \varepsilon\right]}{\mathbb{P}\left[S_{1} \in \mathcal{C}, \ldots, S_{\ell} \in \mathcal{C}, S_{\ell}=g_{\ell}\right]} .
$$

We deduce from the large deviations inequality that there exists a constant $c^{\prime \prime}=c^{\prime \prime}(\varepsilon)>0$ such that for any $k$,

$$
\mathbb{P}\left[\left\|S_{k}-k m\right\| \geq k \varepsilon\right] \leq \exp \left(-c^{\prime \prime} k\right) .
$$

Therefore

$$
\sum_{k \geq b_{\ell}} \mathbb{P}\left[\left\|S_{k}-k m\right\| \geq k \varepsilon\right] \preceq \exp \left(-c^{\prime \prime} b_{\ell}\right) \preceq \exp \left(-c^{\prime \prime} \ell^{\beta}\right)
$$


Since $\alpha<\beta$, Lemma 4.3 .2 and (9) implies that $\lim U_{\ell}=1$. Together with (7) this proves (6). Moreover, the same result holds if we replace $g_{\ell}$ by $g_{\ell}+h_{\ell}$ for $\lim \ell^{-\alpha}\left\|g_{\ell}+h_{\ell}-\ell m\right\|=0$. To achieve the proof of Theorem 4.3.1, it now suffices to establish that

$$
\frac{\mathbb{P}\left[S_{1} \in \mathcal{C}, \ldots, S_{b_{\ell}} \in \mathcal{C}, S_{b_{\ell}} \in B_{b_{\ell}}, S_{\ell}=g_{\ell}+h_{\ell}\right]}{\mathbb{P}\left[S_{1} \in \mathcal{C}, \ldots, S_{b_{\ell}} \in \mathcal{C}, S_{b_{\ell}} \in B_{b_{\ell}}, S_{\ell}=g_{\ell}\right]} \rightarrow 1
$$

Since the increments of the random walk are independent and stationnary, we have

$$
\begin{aligned}
\mathbb{P}\left[S_{1} \in \mathcal{C}, \ldots, S_{b_{\ell}} \in \mathcal{C}, S_{b_{\ell}} \in B_{b_{\ell}},\right. & \left.S_{\ell}=g_{\ell}\right] \\
& =\sum_{x \in B_{b_{\ell}} \cap G} \mathbb{P}\left[S_{\ell-b_{\ell}}=g_{\ell}-x\right] \times \mathbb{P}\left[S_{1} \in \mathcal{C}, \ldots, S_{b_{\ell}} \in \mathcal{C}, S_{b_{\ell}}=x\right]
\end{aligned}
$$

This leads to the theorem since by (5) we have

$$
\mathbb{P}\left[S_{\ell-b_{\ell}}=g_{\ell}-x\right] \sim \mathbb{P}\left[S_{\ell-b_{\ell}}=g_{\ell}+h_{\ell}-x\right],
$$

uniformly in $x \in B_{b_{\ell}}$. (Indeed $\left\|g_{\ell}-x-\left(\ell-b_{\ell}\right) m\right\| \preceq \ell^{\beta} \delta$, uniformly in $x \in B_{b_{\ell}}$.)

\subsection{Renewal theorem}

Assume now that the random variables $X_{\ell}$ take values in a discrete subgroup of $\mathbb{R}^{n}$, and denote by $G$ the group generated by their law $\mu$. We assume that $G$ linearly generates the whole space $\mathbb{R}^{n}$. Denote $U$ the associated renewal measure defined by $U:=\sum_{j \geq 0} \mu^{* j}$. Equivalently, we have for any $g \in G$,

$$
U(g)=\sum_{j=0}^{+\infty} \mathbb{P}\left[S_{j}=g\right] .
$$

Let us first insist that there is no hypothesis of aperiodicity in the following statement ; this is due to the fact that the quantity $U(g)$ represents the expected number of visits of $g$ by the whole random walk and not only at a precise time, like the local limit theorem does. In particular, the law of the variables $X_{l}$ may be supported by a proper coset of $G$ with no consequences on the behavior of $U(g)$ as $g \rightarrow \infty$.

We assume that $m:=\mathbb{E}\left[X_{\ell}\right]$ is nonzero. The renewal theorem tells us that, when $g$ tends to infinity in the direction $m$, we have

$$
U(g) \sim \frac{v(G)}{\sigma}(2 \pi)^{-(n-1) / 2}\|m\|^{(n-3) / 2}\|g\|^{-(n-1) / 2}
$$

where $\sigma^{2}$ is the determinant of the covariance matrix associated to the orthogonal projection of $X_{\ell}$ on the hyperplan orthogonal to $m$. More precisely, we have the following theorem.

Let $\left(e_{1}, e_{2}, \ldots, e_{n-1}\right)$ be an orthonormal basis of the hyperplan $m^{\perp}$. If $x \in \mathbb{R}^{n}$, denote by $x^{\prime}$ its orthogonal projection on $m^{\perp}$ expressed in this basis (here $x^{\prime}$ is regarded as a row vector). Finally let $B$ be the covariance matrix of the random vector $X_{\ell}^{\prime}$.

Recall that $\mathcal{N}_{B}$ is the $(n-1)$-dimensional Gaussian density given by

$$
\mathcal{N}_{B}\left(x^{\prime}\right)=(2 \pi)^{-(n-1) / 2}(\operatorname{det} B)^{-1 / 2} \exp \left(-\frac{1}{2} x^{\prime} \cdot B^{-1} \cdot{ }^{t} x^{\prime}\right) .
$$


Theorem 4.4.1 We assume the random variables $X_{\ell}$ have an exponential moment. Let $\left(a_{\ell}\right)$ be a sequence of real numbers such that $\lim a_{\ell} \ell^{-2 / 3}=0$. Then, when $\ell$ goes to infinity, we have

$$
U(g) \sim \frac{v(G)}{\|m\|} \ell^{-(n-1) / 2} \mathcal{N}_{B}\left(\frac{1}{\sqrt{\ell}} g^{\prime}\right)
$$

uniformly in $g \in G$ such that $\|g-\ell m\| \leq a_{\ell}$.

This theorem has been proved by H. Carlsson and S. Wainger in the case of absolutely continuous distribution (4]). We did not find in the literature the lattice distribution version we state here. A detailed proof of this version is given in [18].

Under the hypotheses of Theorem 4.4.1, we see in particular that if $\left(g_{\ell}\right),\left(h_{\ell}\right)$ are two sequences in $G$ such that $\lim \ell^{-2 / 3}\left\|g_{\ell}-\ell m\right\|=0$ and $\lim \ell^{-1 / 2}\left\|h_{\ell}\right\|=0$, then

$$
U\left(S_{\ell}=g_{\ell}+h_{\ell}\right) \sim U\left(S_{\ell}=g_{\ell}\right) .
$$

\subsection{A quotient renewal theorem for the random walk restricted to a cone}

Theorem 4.5.1 Assume the random variables $X_{\ell}$ are almost surely bounded. Let $\left(g_{\ell}\right),\left(h_{\ell}\right)$ two sequences in $G$ such that there exists $\alpha<2 / 3$ with $\lim \ell^{-\alpha}\left\|g_{\ell}-\ell m\right\|=0$ and $\lim \ell^{-1 / 2}\left\|h_{\ell}\right\|=0$. Then, when $\ell$ tends to infinity, we have

$$
\sum_{j \geq 1} \mathbb{P}\left[S_{1} \in \mathcal{C}, \ldots, S_{j} \in \mathcal{C}, S_{j}=g_{\ell}+h_{\ell}\right] \sim \sum_{j \geq 1} \mathbb{P}\left[S_{1} \in \mathcal{C}, \ldots, S_{j} \in \mathcal{C}, S_{j}=g_{\ell}\right]
$$

Proof. Fix a real number $\beta$ such that $\frac{1}{2}<\alpha<\beta<\frac{2}{3}$ and set $b_{\ell}=\left[\ell^{\beta}\right]$. Let $\delta>0$ be such that $B(m, \delta) \subset \mathcal{C}$ and set $B_{\ell}=B(\ell m, \ell \delta)$. For any $\ell \geq 1$, we have $B_{\ell} \subset \mathcal{C}$.

We are going to prove that

$$
\frac{\sum_{j \geq 1} \mathbb{P}\left[S_{1} \in \mathcal{C}, \ldots, S_{b_{\ell}} \in \mathcal{C}, S_{b_{\ell}} \in B_{b_{\ell}}, S_{j}=g_{\ell}\right]}{\sum_{j \geq 1} \mathbb{P}\left[S_{1} \in \mathcal{C}, \ldots, S_{j} \in \mathcal{C}, S_{j}=g_{\ell}\right]} \rightarrow 1 \quad \text { when } \ell \rightarrow+\infty .
$$

Observe first that there exists $c_{1}>0$ such that $\mathbb{P}\left[S_{j}=g_{\ell}\right]=0$ if $j<c_{1} \ell$ since the support of $\mu$ is bounded.

We prove first that

$$
T_{\ell}:=\frac{\sum_{j \geq c_{1} \ell} \mathbb{P}\left[S_{1} \in \mathcal{C}, \ldots, S_{j} \in \mathcal{C}, S_{b_{\ell}} \in B_{b_{\ell}}, S_{j}=g_{\ell}\right]}{\sum_{j \geq c_{1} \ell} \mathbb{P}\left[S_{1} \in \mathcal{C}, \ldots, S_{j} \in \mathcal{C}, S_{j}=g_{\ell}\right]} \rightarrow 1 \quad \text { when } \ell \rightarrow+\infty
$$

We have

$$
0 \leq 1-T_{\ell} \leq \frac{\sum_{j \geq c_{1} \ell} \mathbb{P}\left[S_{1} \in \mathcal{C}, \ldots, S_{j} \in \mathcal{C}, S_{b_{\ell}} \notin B_{b_{\ell}}, S_{j}=g_{\ell}\right]}{\sum_{j \geq c_{1} \ell} \mathbb{P}\left[S_{1} \in \mathcal{C}, \ldots, S_{j} \in \mathcal{C}, S_{j}=g_{\ell}\right]}
$$

Write $N_{\ell}$ and $D_{\ell}$ for the numerator and the denominator of the previous fraction. We have

$$
D_{\ell} \geq \mathbb{P}\left[S_{1} \in \mathcal{C}, \ldots, S_{\ell} \in \mathcal{C}, S_{\ell}=g_{\ell}\right] .
$$


By Lemma 4.3.2, there exists $c^{\prime}>0$ such that $D_{\ell} \geq \exp \left(-c^{\prime} \ell^{\alpha}\right)$. Since the support of $\mu$ is bounded, there exists $c_{2}>0$ such that $\left|S_{b_{\ell}}\right| \leq c_{2} \ell^{\beta}$. This gives

$$
\begin{aligned}
N_{\ell} \leq \sum_{j \geq c_{1} \ell} \mathbb{P}\left[S_{b_{\ell}}\right. & \left.\notin B_{b_{\ell}}, S_{j}=g_{\ell}\right] \leq \sum_{j \geq c_{1} \ell} \mathbb{P}\left[S_{b_{\ell}} \notin B_{b_{\ell}}\right] \max _{|x| \leq c_{2} \ell^{\beta}} \mathbb{P}\left[x+S_{j-b_{\ell}}=g_{\ell}\right] \\
& \leq \mathbb{P}\left[S_{b_{\ell}} \notin B_{b_{\ell}}\right] \sum_{j \geq c_{1} \ell} \sum_{|x| \leq c_{2} \ell^{\beta}} \mathbb{P}\left[x+S_{j-b_{\ell}}=g_{\ell}\right] \leq \mathbb{P}\left[S_{b_{\ell}} \notin B_{b_{\ell}}\right] \sum_{|x| \leq c_{2} \ell^{\beta}} U\left(g_{\ell}-x\right) .
\end{aligned}
$$

The cardinality of $B\left(0, c_{2} \ell^{\beta}\right) \cap G$ is $O\left(\ell^{n \beta}\right)$ and the function $U$ is uniformly bounded on $G$. This gives

$$
N_{\ell} \preceq \mathbb{P}\left[S_{b_{\ell}} \notin B_{b_{\ell}}\right] \times \ell^{n \beta} .
$$

By the large deviations inequality, there exists $c_{3}>0$ such that $\mathbb{P}\left[S_{b_{\ell}} \notin B_{b_{\ell}}\right] \leq \exp \left(-c_{3} b_{\ell}\right)$. Finally the polynomial term is absorbed by the exponential term and we obtain

$$
N_{\ell} \preceq \exp \left(-c_{4} \ell^{\beta}\right) .
$$

The estimates we have obtained on $D_{\ell}$ et $N_{\ell}$ clearly yields (12).

Next, we prove that

$$
T_{\ell}^{\prime}:=\frac{\sum_{j \geq c_{1} \ell} \mathbb{P}\left[S_{1} \in \mathcal{C}, \ldots, S_{j} \in \mathcal{C}, S_{b_{\ell}} \in B_{b_{\ell}}, S_{j}=g_{\ell}\right]}{\sum_{j \geq c_{1} \ell} \mathbb{P}\left[S_{1} \in \mathcal{C}, \ldots, S_{b_{\ell}} \in \mathcal{C}, S_{b_{\ell}} \in B_{b_{\ell}}, S_{j}=g_{\ell}\right]} \rightarrow 1 \quad \text { when } \ell \rightarrow+\infty .
$$

We have

$$
0 \leq 1-T_{\ell}^{\prime} \leq \frac{\sum_{j \geq c_{1} \ell} \mathbb{P}\left[S_{1} \in \mathcal{C}, \ldots, S_{b_{\ell}} \in \mathcal{C}, S_{b_{\ell}} \in B_{b_{\ell}}, \exists k \in\left\{1, \ldots, j-b_{\ell}\right\} S_{b_{\ell}+k} \notin \mathcal{C}, S_{j}=g_{\ell}\right]}{\sum_{j \geq c_{1} \ell} \mathbb{P}\left[S_{1} \in \mathcal{C}, \ldots, S_{b_{\ell}} \in \mathcal{C}, S_{b_{\ell}} \in B_{b_{\ell}}, S_{j}=g_{\ell}\right]}
$$

Write $N_{\ell}^{\prime}$ and $D_{\ell}^{\prime}$ for the numerator and the denominator of the previous fraction. Let $\varepsilon>0$ be as in the proof of Theorem 4.3.1, Since $\left[S_{k} \notin \mathcal{C}\right] \subset\left[\left\|S_{k}-k m\right\| \geq k \varepsilon\right]$, one gets

$$
\begin{aligned}
N_{\ell}^{\prime} & \leq \sum_{j \geq c_{1} \ell} \mathbb{P}\left[\exists k \in\left\{1, \ldots, j-b_{\ell}\right\} S_{b_{\ell}+k} \notin \mathcal{C}, S_{j}=g_{\ell}\right] \\
& \leq \sum_{k \geq 1} \sum_{j \geq \max \left(c_{1} \ell, k+b_{\ell}\right)} \mathbb{P}\left[S_{b_{\ell}+k} \notin \mathcal{C}, S_{j}=g_{\ell}\right] \\
& \leq \sum_{k \geq 1} \sum_{j \geq k+b_{\ell}} \sum_{y \notin \mathcal{C}} \mathbb{P}\left[S_{b_{\ell}+k}=y\right] \mathbb{P}\left[S_{j-b_{\ell}-k}=g_{\ell}-y\right] \\
& \leq \sum_{k \geq 1} \sum_{y \notin \mathcal{C}} \mathbb{P}\left[S_{b_{\ell}+k}=y\right] U\left(g_{\ell}-y\right) \\
& \leq \sum_{k \geq 1} \sum_{y \notin \mathcal{C}} \mathbb{P}\left[S_{b_{\ell}+k}=y\right] \max _{g \in G} U(g) \\
& \leq \sum_{k \geq b_{\ell}} \mathbb{P}\left[S_{k} \notin \mathcal{C}\right] \max _{g \in G} U(g) \\
& \leq \sum_{k \geq b_{\ell}} \mathbb{P}\left[\left\|S_{k}-k m\right\| \geq k \varepsilon\right] \max _{g \in G} U(g) .
\end{aligned}
$$


Using the large deviations estimate, we obtain as in the proof of Theorem 4.3 .1

$$
N_{\ell}^{\prime} \preceq \exp \left(-c \ell^{\beta}\right) .
$$

Let us now look at the denominator $D_{\ell}^{\prime}$; one gets

$$
D_{\ell}^{\prime} \geq \sum_{j \geq c_{1} \ell} \mathbb{P}\left[S_{1} \in \mathcal{C}, \ldots, S_{j} \in \mathcal{C}, S_{b_{\ell}} \in B_{b_{\ell}}, S_{j}=g_{\ell}\right]
$$

Since $T_{\ell} \rightarrow 1$, we obtain for large enough $\ell$,

$$
D_{\ell}^{\prime} \geq \frac{1}{2} \sum_{j \geq c_{1} \ell} \mathbb{P}\left[S_{1} \in \mathcal{C}, \ldots, S_{j} \in \mathcal{C}, S_{j}=g_{\ell}\right]=D_{\ell}
$$

So $D_{\ell}^{\prime} \geq \frac{1}{2} \exp \left(-c^{\prime} \ell^{\alpha}\right)$ which leads to (13) ; the convergence (11) follows, combining (12) and (13).

The limit (11) also holds when we replace $g_{\ell}$ by $g_{\ell}+h_{\ell}$. Therefore, to prove the theorem, it suffices to check that

$$
\frac{\sum_{j \geq c_{1} \ell} \mathbb{P}\left[S_{1} \in \mathcal{C}, \ldots, S_{b_{\ell}} \in \mathcal{C}, S_{b_{\ell}} \in B_{b_{\ell}}, S_{j}=g_{\ell}+h_{\ell}\right]}{\sum_{j \geq c_{1} \ell} \mathbb{P}\left[S_{1} \in \mathcal{C}, \ldots, S_{b_{\ell}} \in \mathcal{C}, S_{b_{\ell}} \in B_{b_{\ell}}, S_{j}=g_{\ell}\right]} \rightarrow 1
$$

We write the denominator of this fraction as

$$
\sum_{j \geq c_{1} \ell} \sum_{y \in B_{b_{\ell}}} \mathbb{P}\left[S_{1} \in \mathcal{C}, \ldots, S_{b_{\ell}} \in \mathcal{C}, S_{b_{\ell}}=y\right] \times \mathbb{P}\left[S_{j-b_{\ell}}=g_{\ell}-y\right]
$$

which is equal to

$$
\sum_{y \in B_{b_{\ell}}} \mathbb{P}\left[S_{1} \in \mathcal{C}, \ldots, S_{b_{\ell}} \in \mathcal{C}, S_{b_{\ell}}=y\right] \times U\left(g_{\ell}-y\right) .
$$

This is sufficient to conclude since, by(10), we have $U\left(g_{\ell}+h_{\ell}-y\right) \sim U\left(g_{\ell}-y\right)$, uniformly in $y \in B_{b_{\ell}}$.

\section{Crystals and random walks}

\subsection{Brief review on crystals}

We now recall some basics on Kashiwara crystals and quantum groups. For a complete review, we refer to [11] and [13. The quantum group $U_{q}(\mathfrak{g})$ is a $q$-deformation of the enveloping Lie algebra $U(\mathfrak{g})$. To each dominant weight $\lambda \in P_{+}$corresponds a unique (up to isomorphism) irreducible $U_{q}(\mathfrak{g})$-module $V_{q}(\lambda)$. The representation theory of the finite dimensional $U_{q}(\mathfrak{g})$-modules is essentially parallel to that of $U(\mathfrak{g})$. In particular, any tensor product $V_{q}(\lambda) \otimes V_{q}(\mu)$ decomposes into irreducible components. The outer multiplicities so obtained are the same as those appearing in the decomposition of $V(\lambda) \otimes V(\mu)$. Similarly, there exists a relevant notion of weight subspaces in $V_{q}(\lambda)$ and for any $\beta \in P$, one gets $\operatorname{dim} V_{q}(\lambda)_{\beta}=\operatorname{dim} V(\lambda)_{\beta}=K_{\lambda, \beta}$.

To each irreducible module $V_{q}(\lambda)$ is associated its Kashiwara crystal $B(\lambda)$. Formally $B(\lambda):=$ $L(\lambda) / q L(\lambda)$ where $L(\lambda)$ is a particular lattice in $V_{q}(\lambda)$ over the ring

$$
A(q):=\{F \in \mathbb{C}(q) \text { without pole at } q=0\} .
$$


It was proved by Kashiwara that $B(\lambda)$ has the structure of a colored and oriented graph. This graph encodes many informations on the representation $V_{q}(\lambda)$ (and thus also on $V(\lambda)$ ). In particular, the crystal $B(\lambda)$ contains $\operatorname{dim} V_{q}(\lambda)=\operatorname{dim} V(\lambda)$ vertices. Its arrows are labelled by the simple roots $\alpha_{i} \in \Delta_{+}$. The graph structure is obtained from the Kashiwara operators $\tilde{f}_{i}$ and $\tilde{e}_{i}, i \in I$, which are renormalizations of the action of the Chevalley generators $e_{i}, f_{i}$ of $U_{q}(\mathfrak{g})$. More precisely, we have an arrow $a \stackrel{i}{\rightarrow} b$ when $b=\tilde{f}_{i}(a)$ or equivalently $a=\tilde{e}_{i}(b)$. When there is no arrow $\stackrel{i}{\rightarrow}$ starting from $a($ resp. ending at $b)$, we write $\tilde{f}_{i}(a)=0\left(\operatorname{resp} . \tilde{e}_{i}(b)=0\right)$.

The notion of crystal can be extended to a category $\mathcal{O}_{\text {int }}$ of $U_{q}(\mathfrak{g})$ modules containing the irreducible modules $V_{q}(\lambda)$ and stable by tensorization. The crystal associated to the module $M \in$ $\mathcal{O}_{\text {int }}$ is unique up to isomorphism: given $B$ and $B^{\prime}$ two crystals associated to $M$, there exists a bijection $\psi: B \rightarrow B^{\prime}$ which commutes with the Kashiwara operators $\tilde{f}_{i}$ and $\tilde{e}_{i}$.

Given any $b \in B$ and $i \in I$, we set $\varepsilon_{i}(b)=\max \left\{k \mid \tilde{e}_{i}^{k}(b) \neq 0\right\}$ and $\varphi_{i}(b)=\max \left\{k \mid \tilde{f}_{i}^{k}(b) \neq 0\right\}$. The weight of the vertex $b \in B$ is then defined by

$$
\operatorname{wt}(b)=\sum_{i \in I} \operatorname{wt}(b)_{i} \omega_{i} \in P \text { where } \operatorname{wt}(b)_{i}=\varphi_{i}(b)-\varepsilon_{i}(b)
$$

One can then prove that $\operatorname{wt}\left(\tilde{f}_{i}(b)\right)=\operatorname{wt}(b)-\alpha_{i}$ for any $i \in I$ and any $b \in B$ such that $\tilde{f}_{i}(b) \neq 0$. For any $\beta \in P$, the dimension $\operatorname{dim} M_{\beta}$ of the weight space $\beta$ in $M$ is the cardinality of the set $B_{\beta}$ of vertices of weight $\beta$ in the crystal $B$ associated to $M$. A vertex $b \in B$ is said to be of highest weight when $\varepsilon_{i}(b)=0$ for any $i \in I$. In that case, we have immediately that $\operatorname{wt}(b) \in P_{+}$. Write $\mathrm{HW}(B)$ for the set of highest weight vertices in $B$. The elements of $\mathrm{HW}(B)$ are in one-to-one correspondence with the connected components of the crystal $B$. In particular the crystal $B(\lambda)$ is connected with a unique highest weight vertex of weight $\lambda$. For any $b \in B$, we denote by $B(b)$ the connected component of $B$ containing $b$ and by hw $(b)$ the highest weight vertex of $B(b)$.

The two following properties of crystals will be essential for our purpose.

Theorem 5.1.1 Consider $M \in \mathcal{O}_{\text {int }}$ and $B$ its crystal graph.

1. The decomposition of the $U_{q}(\mathfrak{g})$-module $M$ in irreducible components is given be the decomposition of $B$ in connected components. More precisely, we have

$$
M \simeq \bigoplus_{b \in \operatorname{HW}(B)} V_{q}(\mathrm{wt}(b))
$$

2. Consider $\lambda, \mu \in P_{+}$and $B(\lambda), B(\mu)$ the crystals associated to $V_{q}(\lambda)$ and $V_{q}(\mu)$. The crystal associated to $V_{q}(\lambda) \otimes V_{q}(\mu)$ is the crystal $B(\lambda) \otimes B(\mu)$ whose set of vertices is the direct product of the sets of vertices of $B(\lambda)$ and $B(\mu)$ and whose crystal structure is given by the following rules

$$
\tilde{e}_{i}(u \otimes v)=\left\{\begin{array}{l}
u \otimes \tilde{e}_{i}(v) \text { if } \varepsilon_{i}(v)>\varphi_{i}(u) \\
\tilde{e}_{i}(u) \otimes v \text { if } \varepsilon_{i}(v) \leq \varphi_{i}(u)
\end{array} \text { and } \tilde{f}_{i}(u \otimes v)=\left\{\begin{array}{l}
\tilde{f}_{i}(u) \otimes v \text { if } \varphi_{i}(u)>\varepsilon_{i}(v) \\
u \otimes \tilde{f}_{i}(v) \text { if } \varphi_{i}(u) \leq \varepsilon_{i}(v)
\end{array} .\right.\right.
$$

We thus have

$$
\left\{\begin{array}{l}
\varphi_{i}(u \otimes v)=\varphi_{i}(v)+\max \left\{\varphi_{i}(u)-\varepsilon_{i}(v), 0\right\}, \\
\varepsilon_{i}(u \otimes v)=\varepsilon_{i}(u)+\max \left\{\varepsilon_{i}(v)-\varphi_{i}(u), 0\right\} .
\end{array}\right.
$$

In particular $u \otimes v \in \mathrm{HW}(B(\lambda) \otimes B(\mu))$ if and only if $u \in \mathrm{HW}(B(\lambda))$ and $\varepsilon_{i}(v) \leq \varphi_{i}(v)$ for any $i \in I$. 
Example 5.1.2 In type $C_{3}$, the crystal corresponding to the minuscule weight $\omega_{1}$ is

$$
B\left(\omega_{1}\right): 1 \stackrel{1}{\rightarrow} 2 \stackrel{2}{\rightarrow} 3 \stackrel{3}{\rightarrow} \overline{3} \stackrel{2}{\rightarrow} \overline{2} \stackrel{1}{\rightarrow} \overline{1} .
$$

The tensor power $B\left(\omega_{1}\right)^{\otimes 2}$

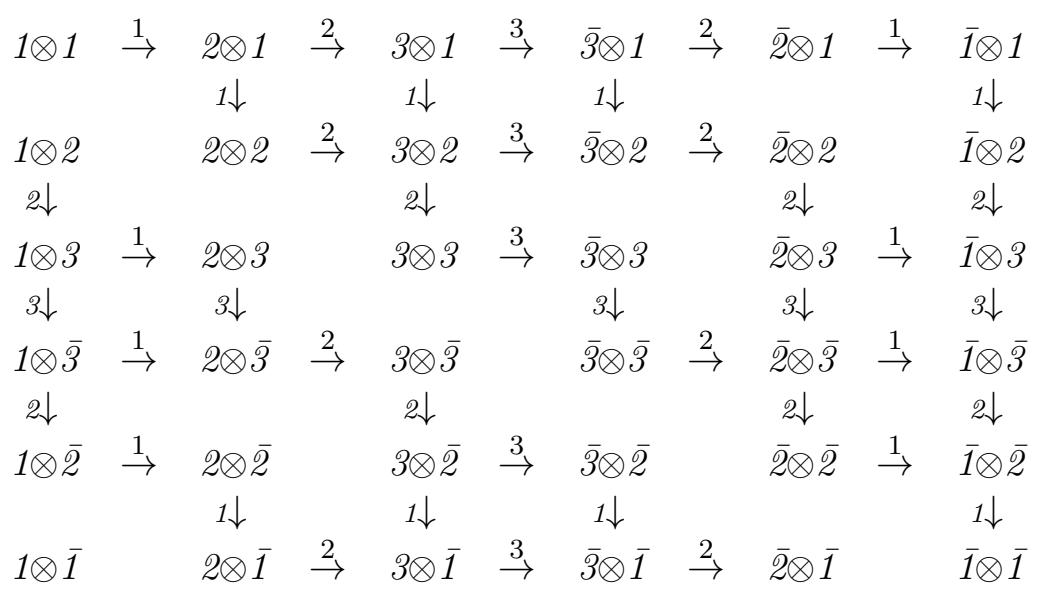

admits three connected components with highest weight vertices $1 \otimes 1,1 \otimes 2$ and $1 \otimes \overline{1}$ of weights $2 \omega_{1}, \omega_{2}$ and 0. This gives the decomposition

$$
V_{q}\left(\omega_{1}\right)^{\otimes 2} \simeq V_{q}\left(2 \omega_{1}\right) \oplus V_{q}\left(\omega_{2}\right) \oplus V_{q}(0) .
$$

Remark: We have seen that $\operatorname{dim} M_{\beta}$ is equal to the number of vertices of weight $\beta$ in $B$ the crystal of $M$. Similarly, the number of highest weight vertices in $B$ with weight $\lambda$ gives the multiplicity of $V(\lambda)$ in the decomposition of $M$ into its irreducible components.

Consider $C$ and $C^{\prime}$ two connected components of the crystal $B$. The components $C$ and $C^{\prime}$ are isomorphic when there exists a bijection $\phi_{C, C^{\prime}}$ from $C$ to $C^{\prime}$ which commutes with the action of the Kashiwara operators, that is $b_{1} \stackrel{i}{\rightarrow} b_{2}$ in $C$ if and only if $\phi_{C, C^{\prime}}\left(b_{1}\right) \stackrel{i}{\rightarrow} \phi_{C, C^{\prime}}\left(b_{2}\right)$ in $C^{\prime}$. In that case, the isomorphism $\phi_{C, C^{\prime}}$ is unique since it must send the highest weight vertex of $C$ on the highest weight vertex of $C^{\prime}$.

The following lemma is a straightforward consequence of Theorem 5.1 .1

Lemma 5.1.3 Assume $b^{0}=a_{1}^{0} \otimes \cdots \otimes a_{\ell}^{0}$ is a highest weight vertex of $B(\delta)^{\otimes \ell}$.

1. For any $k=1, \ldots, \ell$, the vertex $b^{(k), 0}:=a_{1}^{0} \otimes \cdots \otimes a_{k}^{0}$ of $B(\delta)^{\otimes \ell}$ is a highest weight vertex of $B(\delta)^{\otimes k}$ and $\varepsilon_{i}\left(a_{k+1}^{0} \otimes \cdots \otimes a_{\ell}^{0}\right) \leq \varphi_{i}\left(b^{(k), 0}\right)$ for any $i \in I$.

2. Consider $b=a_{1} \otimes \cdots \otimes a_{\ell}$ a vertex of $B\left(b^{0}\right)$. Then, for any $k=1, \ldots, \ell$, the vertex $b^{(k)}:=$ $a_{1} \otimes \cdots \otimes a_{k}$ belongs to $B\left(b^{(k), 0}\right)$.

We will need the following proposition in Section 7 .

Proposition 5.1.4 Consider $\lambda, \mu \in P_{+}$. With the notation of $\S$ 2.2, the following properties hold. 
1. $f_{\lambda / \mu}^{\ell}=\sum_{\kappa \in P_{+}} f_{\kappa}^{\ell} m_{\kappa, \mu}^{\lambda}$ for any $\ell \geq 1$.

2. Assume $\left(\lambda^{(a)}\right)_{a}$ is a sequence of weights of the form $\lambda^{(a)}=a m+o(a)$ with $m \in C$ and consider $\kappa \in P_{+}$. Then, for a sufficiently large, the weight $\lambda^{(a)}$ belongs to $P_{+}$and $m_{\kappa, \mu}^{\lambda^{(a)}}=K_{\mu, \lambda^{(a)}-\kappa}$. Therefore

$$
f_{\lambda^{(a)} / \mu}^{\ell}=\sum_{\kappa \in P_{+}} f_{\kappa}^{\ell} K_{\mu, \lambda(a)-\kappa}=\sum_{\gamma \in P} f_{\lambda^{(a)}-\gamma}^{\ell} K_{\mu, \gamma}
$$

for any $\ell \geq 1$.

Proof. To prove 1, write

$$
s_{\mu}\left(s_{\delta}\right)^{\otimes \ell}=\sum_{\kappa} f_{\kappa}^{\ell} s_{\kappa} s_{\mu}=\sum_{\kappa} \sum_{\lambda} f_{\kappa}^{\ell} m_{\kappa, \mu}^{\lambda} s_{\lambda}=\sum_{\lambda} f_{\lambda / \mu}^{\ell} s_{\lambda}
$$

where all the sums run over $P_{+}$. The assertion immediately follows by comparing the two last expressions.

For $a$ sufficiently large, we must have $\lambda^{(a)} \in P_{+}$for $\lambda^{(a)} \sim a m$ and $m \in C$. By Lemma 5.1.3, $m_{\kappa, \mu}^{\lambda^{(a)}}$ is equal to the number of vertices of weight $\lambda^{(a)}$ of the form $b_{\kappa} \otimes b$ where $b_{\kappa}$ is the highest weight vertex of $B(\kappa)$ and $b \in B(\mu)$ verifies $\varepsilon_{i}(b) \leq \varphi_{i}\left(b_{\kappa}\right)$ for any $i \in I$. In particular $b$ has weight $\lambda^{(a)}-\kappa$. Thus $m_{\kappa, \mu}^{\lambda^{(a)}} \leq K_{\mu, \lambda(a)-\kappa}$.

Now assume $b \in B(\mu)$ has weight $K_{\mu, \lambda}^{(a)}{ }_{-\kappa}$ so that the vertex $b_{\kappa} \otimes b \in B(\kappa) \otimes B(\mu)$ has dominant weight $\lambda^{(a)}$. We have by 2 of Theorem 5.1.1

$$
\mathrm{wt}\left(b_{\kappa} \otimes b\right)_{i}=\varphi_{i}\left(b_{\kappa} \otimes b\right)-\varepsilon_{i}\left(b_{\kappa} \otimes b\right) \leq \varphi_{i}\left(b_{\kappa} \otimes b\right) .
$$

Since $\lambda^{(a)}=a m+o(a)$, the weight $\operatorname{wt}\left(b_{\kappa} \otimes b\right)_{i}$ tends to infinity with $a$, for any $i \in I$. Since $b \in B(\mu)$ and $\mu$ is fixed,

$$
\varphi_{i}\left(b_{k} \otimes b\right)=\varphi_{i}(b)+\max \left\{\varphi_{i}\left(b_{\kappa}\right)-\varepsilon_{i}(b)\right\}>\varphi_{i}(b)
$$

for $a$ sufficiently large. This means that $\varepsilon_{i}(b) \leq \varphi_{i}\left(b_{\kappa}\right)$ for such a $a$. So $b_{\kappa} \otimes b$ is a highest weight vertex with dominant weight $\lambda^{(a)}$. Therefore $m_{\kappa, \mu}^{\lambda^{(a)}} \geq K_{\mu, \lambda^{(a)}-\kappa}$.

\subsection{Paths in weight lattices and crystals}

Let $\delta$ be a dominant weight and $B(\delta)$ the crystal of $V_{q}(\delta)$ 1 1 . By Theorem 5.1.1 and (2), we derive for any $\ell \geq 1$ the decomposition of $B(\delta)^{\otimes \ell}$ in its irreducible components.

$$
B(\delta)^{\otimes \ell} \simeq \bigsqcup_{\lambda \in P_{+}} B(\lambda)^{\oplus f_{\lambda, \delta}^{\ell}}
$$

Let $\mathfrak{P}$ be the map defined on $B(\delta)^{\otimes \ell}$ which associates to each vertex $b$ the highest weight vertex $\mathfrak{P}(b)$ of $B(b)$. The map $\mathfrak{P}$ can be interpreted as a Pitmann transform on paths in the weight lattice following ideas essentially analogue to those used in [1].

Remark: In [19], the transformation $\mathfrak{P}$ was computed by using Knuth insertion algorithm on semistandard tableaux. One can prove that these semistandard tableaux yield simple parametrizations

\footnotetext{
${ }^{1}$ For the sake of simplicity, we only consider in this section paths obtained from tensor powers of irreducible modules. This hypothesis will be relaxed in Section 8 .
} 
of the crystals $B(\delta)$. There exist analogous notions of tableaux for types $B_{n}, C_{n}, D_{n}$ and $G_{2}$ which similarly give a simple parametrization of $B(\delta)$ for any dominant weight $\delta$. They were introduced by Kashiwara and Nakashima in [14] for the classical types and by Kang and Misra for type $G_{2}$ [12]. In [15], [16], 17] one describes combinatorial procedures on these tableaux generalizing Knuth insertion algorithm. They also permit to compute the transformation $\mathfrak{P}$ similarly to the original paper by O'Connell. The computation is then more efficient for it avoids the determination of a path from $b$ to the highest weight vertex $\mathfrak{P}(b)$ of $B(b)$. We do not pursue in this direction and refer to [17] for a simple exposition of these procedures in types $B_{n}, C_{n}, D_{n}$ and $G_{2}$.

To each vertex $b=a_{1} \otimes \cdots \otimes a_{\ell}$ in $B(\delta)^{\otimes \ell}$ naturally corresponds a path in $Z(\delta, \ell)$, namely the path $\left(\mu^{(1)}, \ldots, \mu^{(\ell)}\right)$ where for any $k=1, \ldots, \ell$, we have $\mu^{(k)}=\sum_{j=1}^{k} \operatorname{wt}\left(a_{j}\right)$. We shall denote by $\mathcal{R}$ the map

$$
\mathcal{R}:\left\{\begin{array}{c}
B(\delta)^{\otimes \ell} \rightarrow Z(\delta, \ell) \\
b \mapsto\left(\mu^{(1)}, \ldots, \mu^{(\ell)}\right) .
\end{array}\right.
$$

The map $\mathcal{R}$ is surjective by definition of $Z(\delta, \ell)$. Write $\mathrm{HW}\left(B(\delta)^{\otimes \ell}\right)$ for the set of highest weight vertices in $B(\delta)^{\otimes \ell}$. Then the image of the restriction $\mathcal{R}_{\mathrm{HW}}$ of $\mathcal{R}$ to $\mathrm{HW}\left(B(\delta)^{\otimes \ell}\right)$ is a subset of $Z^{+}(\delta, \ell)$. Indeed, by Lemma 5.1.3, if $b=a_{1} \otimes \cdots \otimes a_{\ell}$ belongs to $\mathrm{HW}\left(B(\delta)^{\otimes \ell}\right)$, then $a_{1} \otimes \cdots \otimes a_{k}$ belongs to $\operatorname{HW}\left(B(\delta)^{\otimes k}\right)$ for any $k=1, \ldots, \ell$.

When each weight space in $V_{q}(\delta)$ has dimension 1 , the map $\mathcal{R}$ is bijective since the steps in the path of $Z(\delta, \ell)$ are in one-to-one correspondence with the weights of $B(\delta)$. We then identify the paths $Z(\delta, \ell)$ with the vertices of $B(\delta)^{\otimes \ell}$. More precisely the path $\left(0, \mu^{(1)}, \ldots, \mu^{(\ell)}\right)$ is identified with the vertex $b=a_{1} \otimes \cdots \otimes a_{\ell} \in B(\delta)^{\otimes \ell}$ where for any $k=1, \ldots, \ell$, the vertex $a_{k}$ is the unique one with weight $\mu^{(k)}-\mu^{(k-1)}$ in $B(\delta)$. This situation happens in particular when $\delta$ is a minuscule weight. Indeed, since each weight in $V_{q}(\delta)$ belongs to the orbit of $\delta$, each weight space has dimension 1. We have the following crucial property.

Proposition 5.2.1 The restriction $\mathcal{R}_{\mathrm{HW}}: \mathrm{HW}\left(B(\delta)^{\otimes \ell}\right) \rightarrow Z^{+}(\delta, \ell)$ is a one-to-one correspondence for any $\ell \geq 1$ if and only if $\delta$ is minuscule.

Proof. Assume $\delta$ is minuscule. We can then identify the paths in $Z^{+}(\delta, \ell)$ with vertices of $B(\delta)^{\otimes \ell}$ as explained above and $\mathcal{R}_{\mathrm{HW}}$ is injective. We thus have to show that any vertex $b=a_{1} \otimes \cdots \otimes a_{\ell} \in$ $B(\delta)^{\otimes \ell}$ such that $\operatorname{wt}\left(a_{1} \otimes \cdots \otimes a_{k}\right)$ is dominant for any $k=1, \ldots, \ell$ is a highest weight vertex. We proceed by induction on $\ell$. For $\ell=1$, the highest weight vertex of $B(\delta)$ is $a_{1}$ for $B(\delta)$ contains a unique vertex of dominant weight ( $\delta$ being minuscule). Assume $b=a_{1} \otimes \cdots \otimes a_{\ell} \in B(\delta)^{\otimes \ell}$ is such that $\operatorname{wt}\left(a_{1} \otimes \cdots \otimes a_{k}\right)$ is dominant for any $k=1, \ldots, \ell$. By the induction hypothesis, $b^{b}=a_{1} \otimes \cdots \otimes a_{\ell-1}$ is of highest weight. According to assertion (2) of Theorem 5.1.1, it suffices to show that $\varepsilon_{i}\left(a_{\ell}\right) \leq \varphi_{i}\left(b^{b}\right)$ for any $i \in I$. But $\varepsilon_{i}\left(a_{\ell}\right) \in\{0,1\}$ since $\delta$ is minuscule. One can therefore assume that $\varepsilon_{i}\left(a_{\ell}\right)=1$. In this case, we have $\varphi_{i}\left(a_{\ell}\right)=0$ because $\delta$ is minuscule. The condition $\operatorname{wt}(b) \in P_{+}$implies that $\varphi_{i}(b) \geq \varepsilon_{i}(b)$ for any $i \in I$. Moreover, the condition wt $(b)=\operatorname{wt}\left(b^{b}\right)+\operatorname{wt}\left(a_{\ell}\right)$ can be written

$$
\varphi_{i}(b)-\varepsilon_{i}(b)=\varphi_{i}\left(b^{b}\right)-\varepsilon_{i}\left(b^{b}\right)+\varphi_{i}\left(a_{\ell}\right)-\varepsilon_{i}\left(a_{\ell}\right)=\varphi_{i}\left(b^{b}\right)-\varepsilon_{i}\left(b^{b}\right)-1 \text { for any } i \in I .
$$

Thus $\varphi_{i}\left(b^{b}\right) \geq \varepsilon_{i}\left(b^{b}\right)+1 \geq \varepsilon_{i}\left(a_{\ell}\right)$ as expected.

Conversely, assume $\mathcal{R}_{\mathrm{HW}}: \mathrm{HW}\left(B(\delta)^{\otimes \ell}\right) \rightarrow Z^{+}(\delta, \ell)$ is a one-to-one correspondence for any $\ell \geq 1$. If $\delta$ is not minuscule, there exists a weight $\mu$ such that $\operatorname{dim} V_{q}(\delta)_{\mu}>0$ which is not in the orbit of $\delta$ under the action of the Weyl group $W$. The orbit of $\mu$ under $W$ intersects the cone of 
dominant weights $P_{+}$. Therefore, we can assume $\mu \in P_{+}$. The crystal $B(\delta)$ thus contains a vertex $b_{\mu}$ of dominant weight $\mu$ which is not a highest weight vertex since $\mu \neq \delta$. Then the path in $P$ from 0 to $\mu$ belongs to $Z^{+}(\delta, 1)$ but is not in the image of $\mathrm{HW}(B(\delta))$ by $\mathcal{R}_{\mathrm{HW}}$ which contradicts our assumption.

Remark: There exist dominant weights $\delta$ which are not minuscule but such that each weight spaces of $V(\delta)$ has dimension 1 . This is notably the case for $\delta=k \omega_{1}, k \in \mathbb{N}$ in type $A_{n-1}$ and $\delta=\omega_{1}$ in type $B_{n}$. In that case, according to the previous proposition, the map $\mathcal{R}_{\mathrm{HW}}$ does not provide a bijection between highest weight vertices and paths in $P_{+}$.

Let $Z^{+}(\delta, \ell, \lambda)$ be the subset of $Z^{+}(\delta, \ell)$ of paths starting at 0 and ending at $\lambda \in P_{+}$. Theorem 5.1 .1 and the previous Proposition immediately yield the following corollary equating the number of paths in $Z^{+}(\delta, \ell, \lambda)$ to an outer multiplicity in the tensor power $V(\delta)^{\otimes \ell}$.

Corollary 5.2.2 Assume $\delta$ is minuscule. We have $\operatorname{card}\left(Z^{+}(\delta, \ell, \lambda)\right)=f_{\lambda, \delta}^{\ell}$ where the outer multiplicity $f_{\lambda, \delta}^{\ell}$ is defined by (2).

As far as we are aware this equality was first established by Grabiber and Mayard in [8] by using some previous works of Proctor [21]. Crystal basis theory permits to derive a very short proof of this identity.

\subsection{Probability distribution on $B(\delta)$}

The aim of this paragraph is to endow $B(\delta)$ with a probability distribution. We are going to associate to each vertex $a \in B(\delta)$ a probability $p_{b}$ such that

$$
0<p_{a}<1 \text { and } \sum_{a \in B(\delta)} p_{a}=1 .
$$

The probability distributions we consider are compatible with the weight graduation, that is for any $a, a^{\prime} \in B(\delta)$,

$$
\operatorname{wt}(a)=\operatorname{wt}\left(a^{\prime}\right) \Longrightarrow p_{a}=p_{a^{\prime}} .
$$

We proceed as follows. Let $t=\left(t_{1}, \ldots, t_{n}\right)$ be a $n$-tuple of positive reals (recall that $n$ is the rank of the root system considered). Let $a \in B(\delta)$. For any $\kappa \in Q_{+}$such that $\kappa=\sum_{i=1}^{n} m_{i} \alpha_{i}$, we set $t^{[\kappa]}=t_{1}^{m_{1}} \cdots t_{n}^{m_{n}}$. Since wt $(a)$ is a weight of $V_{q}(\delta)$, there exist nonnegative integers $m_{1}, \ldots, m_{n}$ such that $\operatorname{wt}(a)=\delta-\sum_{i=1}^{n} m_{i} \alpha_{i}$. We can compute the sum $\Sigma_{\delta}(t)=\sum_{a \in B(\delta)} t^{[\operatorname{wt}(a)]}$. The probability distribution is then defined by

$$
p_{a}=\frac{t^{[\mathrm{wt}(a)]}}{\Sigma_{\delta}(t)} \text { for any } a \in B(\delta),
$$

so that we have $\sum_{a \in B(\delta)} p_{a}=1$. It is clear that conditions (II) and (III) are satisfied. Note that if we have an arrow $a \stackrel{i}{\rightarrow} a^{\prime}$ in $B(\delta)$, then $p_{a^{\prime}}=p_{a} \times t_{i}$ since $\operatorname{wt}\left(a^{\prime}\right)=\operatorname{wt}(a)-\alpha_{i}$. Also, the integer $m_{i}$ can easily be read on the crystal $B(\delta)$. It corresponds to the number of arrows $\stackrel{i}{\rightarrow}$ in any path connected the highest weight vertex of $B(\delta)$ to $a$. Recall that these numbers are independent of the path considered since we have the weight graduation wt on the crystals. The case where $t_{i}=1$ for any $i=1, \ldots, n$, corresponds to the uniform probability distribution on $B(\delta)$ considered in [1]. 
Remark: Observe that our construction depends only on the fixed $n$-tuple $t=\left(t_{1}, \ldots, t_{n}\right)$.

When $t_{1}=\cdots=t_{n}$, one gets $p_{a}=\frac{1}{\operatorname{dim} V(\delta)}$ for any $a \in B(\delta)$. We shall say in this case that the distribution is uniform. In the rest of the paper, we assume the $n$-tuple $t=\left(t_{1}, \ldots, t_{n}\right)$ is fixed for each root system corresponding to a simple Lie algebra. We denote by $x=\left(x_{1}, \ldots, x_{N}\right) \in \mathbb{R}_{>0}^{N}$ any solution of the algebraic system

$$
x^{\alpha_{i}}=t_{i}^{-1}, i=1, \ldots, n \text {. }
$$

Let us briefly explain why such a solution necessary exists. Let $U=\left(u_{i, j}\right)$ be the $N \times n$ matrix whose entries are determined by the decompositions $\alpha_{i}=\sum_{j=1}^{N} u_{i, j} \varepsilon_{j}, i=1, \ldots, n$. By taking the logarithm of each equation in (19), we are lead to solve the equation ${ }^{t} U X=Y$ where $X=\left(\ln x_{1}, \ldots, \ln x_{N}\right)$ and $Y=\left(-\ln t_{1}, \ldots,-\ln t_{n}\right)$. This linear system necessary admits solutions because $U$ has rank $n$. This follows from the fact that the set of simple roots $\left\{\alpha_{1}, \ldots, \alpha_{n}\right\}$ generates a $n$-dimensional subspace in $\mathbb{R}^{N}$. Each solution $X$ yields positive solutions $x_{1}, \ldots, x_{N}$ of (19). Observe that (19) admits a unique solution when $N=n$. With the convention of [3], this happens for the root systems $B_{n}, C_{n}, D_{n}, E_{8}, F_{4}$ and $G_{2}$. Now consider $a \in B(\delta)$. By the previous definition of $t_{a}$, we then derive the relation $t_{a}=x^{-\delta} x^{\mathrm{wt}(a)}$. This allows to write

$$
\Sigma_{\delta}(t)=x^{-\delta} \sum_{a \in B(\delta)} x^{\mathrm{wt}(a)}=x^{-\delta} s_{\delta}(x) \text { and } p_{a}=\frac{x^{\mathrm{wt}(a)}}{s_{\delta}(x)} .
$$

\section{Example 5.3.1}

1. Assume $\delta=\omega_{1}$ for type $A_{n-1}$. Then we have

$$
B(\delta): a_{1} \stackrel{1}{\rightarrow} a_{2} \stackrel{2}{\rightarrow} \cdots \stackrel{n-1}{\rightarrow} a_{n}
$$

The simple roots are the $\alpha_{i}=\varepsilon_{i}-\varepsilon_{i+1}, i=1, \ldots, n-1$. We thus have $t_{i}=\frac{x_{i+1}}{x_{i}}$ for any $i=1, \ldots, n-1$. We obtain $x_{i}=x_{1} t_{1} \cdots t_{i-1}$ for any $i=2, \ldots, n$. In that case, we have $N=(n-1)+1$ where $n-1$ is the rank of the root system considered. We can normalize our probability distribution so that $x_{1}+\cdots+x_{n}=1$. This gives $p_{i}=x_{i}$ for $i=1, \ldots, n$. The corresponding random walk in $P$ corresponds to the ballot problem where each transition in the direction $\varepsilon_{i}$ has probability $p_{i}$.

2. Assume $\delta=\omega_{1}$ for type $C_{n}$. Then we have

$$
B(\delta): a_{1} \stackrel{1}{\rightarrow} a_{2} \stackrel{2}{\rightarrow} \cdots \stackrel{n-1}{\rightarrow} a_{n} \stackrel{1}{\rightarrow} a_{\bar{n}} \stackrel{n-1}{\rightarrow} \cdots \stackrel{2}{\rightarrow} a_{\overline{2}} \stackrel{1}{\rightarrow} a_{\overline{1}} .
$$

The simple roots are the $\alpha_{i}=\varepsilon_{i}-\varepsilon_{i+1}, i=1, \ldots, n-1$ and $\alpha_{n}=2 \varepsilon_{n}$. We thus have $t_{i}=\frac{x_{i+1}}{x_{i}}$ for any $i=1, \ldots, n-1$ and $t_{n}=x_{n}^{-2}$. This gives $x_{i}=\frac{1}{t_{i} \cdots t_{n-1} \sqrt{t_{n}}}$ for any $i=1, \ldots n-1$ and $x_{n}=\frac{1}{\sqrt{t_{n}}}$. So $p_{a_{i}}=\frac{x_{i}}{s_{\omega_{1}}(x)}$ and $p_{a_{\bar{i}}}=\frac{x_{i}^{-1}}{s_{\omega_{1}}(x)}$ where $s_{\omega_{1}}(x)=\sum_{i=1}^{n}\left(x_{i}+x_{i}^{-1}\right)$. We thus obtain a random walk in $\mathbb{Z}^{n}$, with steps from one point to one of the nearest neighbors, and $p_{a_{i}} p_{a_{\bar{i}}}$ independent of $i$. 


\subsection{The random walk $\mathcal{W}$ in the weight lattice}

\subsubsection{From a probability measure on the crystal $B(\delta)$ to a random walk on the weight lattice.}

A random walk in the weight lattice $P$ isomorphic to $\mathbb{Z}^{n}$ is characterized by the law of its increments, that is a probability measure on $P$. As the map $b \mapsto \operatorname{wt}(b)$ sends the crystal $B(\delta)$ into $P$, any probability measure $p$ on $B(\delta)$ can be pushed forward and defined a probability $\mathrm{wt}_{*} p$ on $P$.

The random walk with law $\mathrm{wt}_{*} p$ can be naturally realized as a random process defined on the infinite product space $\mathcal{B}(\delta)^{\otimes \mathbb{N}}$ equipped with the product measure $p^{\otimes \mathbb{N}}$. Here is a "concrete" realization of this construction. The infinite product space $\mathcal{B}(\delta)^{\otimes \mathbb{N}}$ is the projective limit of the sequence of tensor products $\left(B(\delta)^{\otimes \ell}\right)_{\ell \in \mathbb{N}}$ associated to the projections $\pi_{\ell^{\prime}, \ell}\left(a_{1} \otimes a_{2} \otimes \ldots \otimes a_{\ell^{\prime}}\right)=$ $a_{1} \otimes a_{2} \otimes \ldots \otimes a_{\ell}$ if $\ell \leq \ell^{\prime}$. We denote by $\pi_{\infty, \ell}$ the canonical projection from $\mathcal{B}(\delta)^{\otimes \mathbb{N}}$ onto $B(\delta)^{\otimes \ell}$. By Kolmogorov's theorem, we know that there exists a unique probability measure $\mathbb{P}$ on the space $\mathcal{B}(\delta)^{\otimes \mathbb{N}}$ whose image by each projection $\pi_{\infty, \ell}$ is the probability $\mathbb{P}^{(\ell)}$ defined by

$$
\mathbb{P}^{(\ell)}\left(a_{1} \otimes a_{2} \otimes \ldots \otimes a_{\ell}\right)=\prod_{i=1}^{\ell} p\left(a_{i}\right) .
$$

On the probability space $\left(\mathcal{B}(\delta)^{\otimes \mathbb{N}}, \mathbb{P}\right)$, the random variables

$$
a_{1} \otimes a_{2} \otimes \ldots \mapsto a_{\ell}, \quad \ell \in \mathbb{N}
$$

are independent and identically distributed with law $p$.

Now, on this probability space we define the random variables $\mathcal{W}_{\ell}$ by

$$
\mathcal{W}_{\ell}=\mathrm{wt} \circ \pi_{\infty, \ell}
$$

that is

$$
\mathcal{W}_{\ell}(b)=\mathrm{wt}\left(b^{(\ell)}\right)
$$

if $b=a_{1} \otimes a_{2} \otimes \ldots$ and $b^{(\ell)}=a_{1} \otimes a_{2} \otimes \ldots \otimes a_{\ell}$.

The random process $\left(\mathcal{W}_{\ell}\right)_{\ell \in \mathbb{N}}$ is a realization of the random walk on $P$ with law wt $p$. Indeed, for any $\beta, \beta^{\prime} \in P$, we have

$$
\begin{aligned}
\mathbb{P}\left(\mathcal{W}_{\ell+1}=\beta^{\prime}\right. & \left.\mid \mathcal{W}_{\ell}=\beta\right)=\mathbb{P}^{(\ell+1)}\left(\operatorname{wt}\left(b^{(\ell)} \otimes a_{\ell+1}\right)=\beta^{\prime} \mid \operatorname{wt}\left(b^{(\ell)}\right)=\beta\right)= \\
& \mathbb{P}^{(\ell+1)}\left(\operatorname{wt}\left(b^{(\ell)}\right)+\operatorname{wt}\left(a_{\ell+1}\right)=\beta^{\prime} \mid \operatorname{wt}\left(b^{(\ell)}\right)=\beta\right)=\mathbb{P}^{(\ell+1)}\left(\operatorname{wt}\left(a_{\ell+1}\right)=\beta^{\prime}-\beta\right) .
\end{aligned}
$$

\subsubsection{Application to our particular situation}

Now we come back to the particular choice of a probability distribution $p$ on the crystal $B(\delta)$ which has been described in the previous section ; the probability of a vertex $b$ depends only on its weight and, if there is an arrow $b \stackrel{i}{\rightarrow} b^{\prime}$, then $p_{b^{\prime}}=p_{b} \times t_{i}$.

We extend the notation $p_{b}$ to the vertices $b \in B(\delta)^{\otimes \ell}$ using the rule (20) :

$$
p_{a_{1} \otimes a_{2} \otimes \ldots \otimes a_{\ell}}=p_{a_{1}} p_{a_{2}} \ldots p_{a_{\ell}} .
$$


We recall that if $b \in B(\delta)$, then $p_{b}=\frac{x^{\mathrm{wt}(b)}}{s_{\delta}(x)}$. We see that if $b \in B(\delta)^{\otimes \ell}$, then

$$
p_{b}=\frac{x^{\mathrm{wt}(b)}}{s_{\delta}(x)^{\ell}} .
$$

Using the rules of construction of the tensor powers of the crystal graph, it is straightforward to verify that the previous properties extend to tensor powers : let $b, b^{\prime}$ in $B(\delta)^{\otimes \ell}$,

- Assume we have $\operatorname{wt}(b)=\operatorname{wt}\left(b^{\prime}\right)$. Then $p_{b}=p_{b^{\prime}}$.

- Assume we have an arrow $b \stackrel{i}{\rightarrow} b^{\prime}$ in $B(\delta)^{\otimes \ell}$. Then $p_{b^{\prime}}=p_{b} \times t_{i}$.

If we follow the construction described in Subsection 5.4.1, we obtain a random walk $\left(\mathcal{W}_{\ell}\right)_{\ell \in \mathbb{N}}$ on the weight lattice $P$ with the following properties :

- the law of the increments of the random walk $\left(\mathcal{W}_{\ell}\right)$ is given by

$$
\mathbb{P}\left(\mathcal{W}_{\ell+1}=\beta^{\prime} \mid \mathcal{W}_{\ell}=\beta\right)=\frac{K_{\delta, \beta^{\prime}-\beta} x^{\beta^{\prime}-\beta}}{s_{\delta}(x)}
$$

- the expectation of the increments, also called the drift of the random walk, is

$$
m:=\frac{1}{s_{\delta}(x)} \sum_{\alpha} K_{\delta, \alpha} x^{\alpha} \alpha
$$

- the probability of a finite path $\pi=\left(\beta_{0}=0, \beta_{1}, \beta_{2}, \ldots, \beta_{\ell}\right)$ is given by

$$
\frac{1}{s_{\delta}(x)^{\ell}} K_{\delta, \alpha^{(1)}} \times \cdots \times K_{\delta, \alpha^{(\ell)}} x^{\beta_{\ell}}
$$

where $\alpha^{(i)}=\beta_{i}-\beta_{i-1}$ for any $i=1, \ldots, \ell$.

\section{Remarks:}

1. Consider a dominant weight $\lambda$ and $B$ a connected component in $B(\delta)^{\otimes \ell}$ isomorphic to $B(\lambda)$. We set

$$
S_{\lambda, \ell}(x):=\sum_{b \in B} p_{b}=\sum_{\beta \in P} \sum_{b \in B_{\beta}} \frac{x^{\beta}}{s_{\delta}(x)^{\ell}}=\frac{1}{s_{\delta}(x)^{\ell}} \sum_{\beta \in P} K_{\lambda, \beta} x^{\beta}=\frac{s_{\lambda}(x)}{s_{\delta}(x)^{\ell}}
$$

where the second equality is a consequence of (22) and the last equality is obtained by definition of the Weyl character. Clearly, $S_{\lambda, \ell}(x)$ gives the probability $\mathbb{P}(B)$ that a random vertex of $B(\delta)^{\otimes \ell}$ belongs to $B$. Observe that this probability does not depend on the connected component $B$ itself but only on $\lambda$ and $\ell$.

2. When $\lambda=\delta$ is minuscule the situation simplifies. Indeed $K_{\delta, \alpha}=1$ for any weight $\alpha$ of $V(\delta)$, therefore the map wt is one-to-one on $B(\delta)$. The probability of the path $\pi=$ $\left(\beta_{0}=0, \beta_{1}, \beta_{2}, \ldots, \beta_{\ell}\right)$ is then equal to $\frac{x^{\beta} \ell}{s_{\delta}(x)^{\ell}}$. In particular, when $\delta$ is minuscule, two paths starting and ending at the same points have the same probability. 


\section{Markov chains in the Weyl chamber}

The purpose of this section is to introduce a Markov chain $\left(\mathcal{H}_{\ell}\right)$ in the Weyl chamber obtained from $\left(\mathcal{W}_{\ell}\right)_{\ell \geq 1}$ by an operation on crystals. This operation consists in the composition of $\mathcal{W}_{\ell}$ with a transformation of $B(\delta)^{\otimes \mathbb{N}}$ which plays the role of the Pitman transformation. Here and in the sequel, $\delta$ is a fixed dominant weight, i.e. an element of $P_{+}$.

\subsection{The Markov chain $\mathcal{H}$}

The map $\mathfrak{P}$ has been introduced in $\S 2.4$. It associates to any vertex $b(\ell) \in B(\delta)^{\otimes \ell}$, the highest weight vertex $\mathfrak{P}(b(\ell))$ of $B(b(\ell)) \subset B(\delta)^{\otimes \ell}$. By Lemma 5.1.3, the transformation on $B(\delta)^{\otimes \mathbb{N}}$ also denoted by $\mathfrak{P}$ such that $\mathfrak{P}(b)=(\mathfrak{P}(b(\ell)))_{\ell \geq 1}$ for any $b=(b(\ell))_{\ell \geq 1} \in B(\delta)^{\otimes \mathbb{N}}$ is well-defined. We then consider the random variable $\mathcal{H}_{\ell}:=\mathcal{W}_{\ell} \circ \mathfrak{P}$ (see (21)) defined on the probability space $\Omega(\delta)=\left(B(\delta)^{\otimes \mathbb{N}}, \mathbb{P}\right)$ with values in $P_{+}$. This yields a stochastic process $\mathcal{H}=\left(\mathcal{H}_{\ell}\right)_{\ell \geq 0}$.

Proposition 6.1.1 Consider $\ell \in \mathbb{Z}$ and $\lambda \in P_{+}$. Then $\mathbb{P}\left(\mathcal{H}_{\ell}=\lambda\right)=f_{\lambda, \delta}^{\ell} \frac{s_{\lambda}(x)}{s_{\delta}(x)^{\ell}}$.

Proof. By definition of the random variable $\mathcal{H}_{\ell}$, we have

$$
\mathbb{P}\left(\mathcal{H}_{\ell}=\lambda\right)=\sum_{b_{\lambda} \in \mathrm{HW}\left(B(\delta)^{\otimes \ell}\right), \operatorname{wt}\left(b_{\lambda}\right)=\lambda} \mathbb{P}\left(B\left(b_{\lambda}\right)\right) .
$$

We have seen in (25) that $\mathbb{P}\left(B\left(b_{\lambda}\right)\right)=\frac{s_{\lambda}(x)}{s_{\delta}(x)^{\ell}}$ does not depend on $b_{\lambda}$ but only on $\lambda$. By definition of $f_{\lambda, \delta}^{\ell}$ (see (2) ) and Theorem 5.1.1, the number of connected components in $B(\delta)^{\otimes \ell}$ isomorphic to $B(\lambda)$ is equal to $f_{\lambda, \delta}^{\ell}$. This gives $\mathbb{P}\left(\mathcal{H}_{\ell}=\lambda\right)=f_{\lambda, \delta}^{\ell} \frac{s_{\lambda}(x)}{s_{\delta}(x)^{\ell}}$.

We can now state the main result of this Section

Theorem 6.1.2 The stochastic process $\mathcal{H}$ is a Markov chain with transition probabilities

$$
\Pi_{\mathcal{H}}(\mu, \lambda)=\frac{m_{\mu, \delta}^{\lambda} s_{\lambda}(x)}{s_{\delta}(x) s_{\mu}(x)} \quad \lambda, \mu \in P_{+} .
$$

Proof. Consider a sequence of dominant weights $\lambda^{(1)}, \ldots, \lambda^{(\ell)}, \lambda^{(\ell+1)}$ such that $\lambda^{(1)}=\delta, \lambda^{(\ell)}=\mu$ and $\lambda^{(\ell+1)}=\lambda$. Recall that, for any $k=1, \ldots, \ell$, the integer $m_{\lambda^{(k)}, \delta}^{\lambda^{(k+1)}}$ is the multiplicity of $V\left(\lambda^{(k+1)}\right)$ in $V\left(\lambda^{(k)}\right) \otimes V(\delta)$. Let $b \in \Omega(\delta)$ be such that $\mathcal{H}_{k}(b)=\lambda^{(k)}$ for any $k=1, \ldots, \ell+1$. Write $h^{(\ell+1)}=a_{1}^{h} \otimes \cdots \otimes a_{\ell+1}^{h}$ for the highest weight vertex of $B\left(b^{(\ell+1)}\right) \subset B(\delta)^{\otimes \ell+1}$. By Lemma 5.1.3, for $k=1, \ldots, \ell+1$, the vertex $h^{(k)}=a_{1}^{h} \otimes \cdots \otimes a_{k}^{h}$ is the highest weight vertex of $b^{(k)}$ and has weight $\lambda^{(k)}$. Let us denote by $S$ the set of highest weight vertices of $B(\delta)^{\otimes \ell+1}$ whose projection on $B(\delta)^{k}$ has weight $\lambda^{(k)}$ for any $k=1, \ldots, \ell+1$. By Theorem 5.1.1 and a straightforward induction, we have

$$
\operatorname{card}(S)=\prod_{k=1}^{\ell} m_{\lambda^{(k), \delta}}^{\lambda^{(k+1)}} .
$$

We can now write

$$
\mathbb{P}\left(\mathcal{H}_{\ell+1}=\lambda, \mathcal{H}_{k}=\lambda^{(k)} \text { for any } k=1, \ldots, \ell\right)=\sum_{h \in S} \sum_{b \in B(h)} p_{b}=\sum_{h \in S} \mathbb{P}(B(h)) .
$$


Each $h$ in $S$ is a highest weight vertex of weight $\lambda$ and one gets $\mathbb{P}(B(h))=S_{\lambda, \ell+1}(x)$ (see (25)). This gives

$$
\mathbb{P}\left(\mathcal{H}_{\ell+1}=\lambda, \mathcal{H}_{k}=\lambda^{(k)} \text { for any } k=1, \ldots, \ell\right)=\prod_{k=1}^{\ell} m_{\lambda^{(k)}, \delta}^{\lambda^{(k+1)}} \times S_{\lambda, \ell+1}(x) .
$$

Similarly, we have

$$
\mathbb{P}\left(\mathcal{H}_{k}=\lambda^{(k)} \text { for any } k=1, \ldots, \ell\right)=\prod_{k=1}^{\ell-1} m_{\lambda^{(k)}, \delta}^{\lambda^{(k+1)}} \times S_{\mu, \ell}(x) .
$$

Hence

$$
\mathbb{P}\left(\mathcal{H}_{\ell+1}=\lambda \mid \mathcal{H}_{k}=\lambda^{(k)} \text { for any } k=1, \ldots, \ell\right)=\frac{m_{\lambda^{(\ell)}, \delta}^{\lambda^{(\ell+1)}} S_{\lambda^{(\ell+1)}, \ell+1}(x)}{S_{\lambda^{(\ell)}, \ell}(x)}=\frac{m_{\mu, \delta}^{\lambda} s_{\lambda}(x)}{s_{\delta}(x) s_{\mu}(x)} .
$$

In particular, $\mathbb{P}\left(\mathcal{H}_{\ell+1}=\lambda \mid \mathcal{H}_{k}=\lambda^{(k)}\right.$ for any $\left.k=1, \ldots, \ell\right)$ depends only on $\lambda$ and $\mu=\lambda^{(\ell)}$ which shows the Markov property.

\subsection{Intertwining operators}

For any $\lambda \in P_{+}$and $\beta \in P$, the event $\left(\mathcal{W}_{\ell}=\beta, \mathcal{H}_{\ell}=\lambda\right)$ contains all the elements $b$ in $\Omega(\delta)$ such that $b^{(\ell)}$ has weight $\beta$ and belongs to a connected component of $B(\delta)^{\otimes \ell}$ with highest weight $\lambda$. This gives $\mathbb{P}\left(\mathcal{W}_{\ell}=\beta, \mathcal{H}_{\ell}=\lambda\right)=\frac{1}{s_{\delta}(x)^{\ell}} f_{\ell, \lambda} K_{\lambda, \beta} x^{\beta}$ since there is $f_{\ell, \lambda}$ connected components in $B(\delta)^{\otimes \ell}$ of highest weight $\lambda$, each of them contains $K_{\lambda, \beta}$ vertices of weight $\beta$ whose common probability is $\frac{x^{\beta}}{s_{\delta}(x)^{\ell}}$. Using Proposition 6.1.1, we obtain

$$
\mathbb{P}\left(\mathcal{W}_{\ell}=\beta \mid \mathcal{H}_{\ell}=\lambda\right)=\frac{K_{\lambda, \beta} x^{\beta}}{s_{\lambda}(x)}
$$

which is independent of $\ell$. We set $\mathcal{K}(\lambda, \beta):=\mathbb{P}\left(\mathcal{W}_{\ell}=\beta \mid \mathcal{H}_{\ell}=\lambda\right)=\frac{K_{\lambda, \beta} x^{\beta}}{s_{\lambda}(x)}$.

Theorem 6.2.1 We have the intertwining relation $\Pi_{\mathcal{H}} \mathcal{K}=\mathcal{K} \Pi_{\mathcal{W}}$.

Proof. Consider $\mu \in P_{+}$and $\beta \in P$. Write $\left(\Pi_{\mathcal{H}} \mathcal{K}\right)(\mu, \beta)$ for the coefficient of the matrix product $\Pi_{\mathcal{H}} \mathcal{K}$ associated to the pair $(\mu, \beta)$. Define $\left(\mathcal{K} \Pi_{\mathcal{W}}\right)(\mu, \beta)$ similarly. By using Theorem 6.1.2 and (26), we have

$$
\left(\Pi_{\mathcal{H}} \mathcal{K}\right)(\mu, \beta)=\sum_{\lambda \in P_{+}} \Pi_{\mathcal{H}}(\mu, \lambda) \mathcal{K}(\lambda, \beta)=\sum_{\lambda \in P_{+}} \frac{m_{\mu, \delta}^{\lambda} s_{\lambda}(x)}{s_{\delta}(x) s_{\mu}(x)} \frac{K_{\lambda, \beta} x^{\beta}}{s_{\lambda}(x)}=\sum_{\lambda \in P_{+}} \frac{m_{\mu, \delta}^{\lambda} K_{\lambda, \beta} x^{\beta}}{s_{\delta}(x) s_{\mu}(x)} .
$$

This gives

$$
\left(\Pi_{\mathcal{H}} \mathcal{K}\right)(\mu, \beta)=\frac{x^{\beta}}{s_{\delta}(x) s_{\mu}(x)} \sum_{\lambda \in P_{+}} m_{\mu, \delta}^{\lambda} K_{\lambda, \beta}
$$

On the other hand, using (23)

$$
\left(\mathcal{K} \Pi_{\mathcal{W}}\right)(\mu, \beta)=\sum_{\gamma \in P} \mathcal{K}(\mu, \gamma) \Pi_{\mathcal{W}}(\gamma, \beta)=\sum_{\gamma \in P} \frac{K_{\mu, \gamma} x^{\gamma}}{s_{\mu}(x)} K_{\delta, \beta-\gamma} \frac{x^{\beta-\gamma}}{s_{\delta}(x)}
$$


This yields to $\left(\mathcal{K} \Pi_{\mathcal{W}}\right)(\mu, \beta)=\frac{x^{\beta}}{s_{\delta}(x) s_{\mu}(x)} \sum_{\gamma \in P} K_{\mu, \gamma} K_{\delta, \beta-\gamma}$. Therefore the equality $\Pi_{\mathcal{H}} \mathcal{K}=\mathcal{K} \Pi_{\mathcal{W}}$ reduces to $\sum_{\lambda \in P_{+}} m_{\mu, \delta}^{\lambda} K_{\lambda, \beta}=\sum_{\gamma \in P} K_{\mu, \gamma} K_{\delta, \beta-\gamma}$ which was established in Lemma 2.2.1.

\section{Restriction to the Weyl chamber}

We have explicit formulae for the transition matrices $\Pi_{\mathcal{W}}$ and $\Pi_{\mathcal{H}}$ of the Markov chains $\mathcal{W}$ and $\mathcal{H}$. The matrix $\Pi_{\mathcal{W}}$ has entries in $P$ and the matrix $\Pi_{\mathcal{H}}$ has entries in $P_{+}$. We will see that if the representation $\delta$ is minuscule then $\Pi_{\mathcal{H}}$ is a Doob transform of the restriction of $\Pi_{\mathcal{W}}$ to $P_{+}$.

\subsection{Doob transform of the random walk $\mathcal{W}$ restricted to the Weyl chamber}

Recall that $\left(\mathcal{W}_{\ell}\right)_{\ell \geq 1}$ is a Markov chain with transition matrix $\Pi_{\mathcal{W}}$. Since the closed Weyl chamber $\bar{C}$ is a subset of $\mathbb{R}^{N}$, it makes sense to consider the substochastic matrix $\Pi_{\mathcal{W}}^{\bar{C}}(\mu, \lambda)$, that is the restriction of the transition matrix of $\mathcal{W}$ to $\bar{C}$. The following Proposition answers the question whether the transition matrix $\Pi_{\mathcal{H}}$ of the Markov chain $\mathcal{H}$ (see Theorem 6.1.2) can be regarded as a Doob transform of $\Pi_{\mathcal{W}}^{\bar{C}}$. We denote by $\psi$ the function defined on $P_{+}$by $\psi(\lambda)=x^{-\lambda} s_{\lambda}(x)$.

Proposition 7.1.1 If $\delta$ is a minuscule representation, then the transition matrix $\Pi_{\mathcal{H}}$ is the Doob $\psi$-transform of the substochastic matrix $\Pi_{\mathcal{W}}^{C}$, in particular $\psi$ is harmonic with respect to this substochastic matrix. If $\delta$ is not minuscule, then the transition matrix $\Pi_{\mathcal{H}}$ cannot be realized as a Doob transform of the substochastic matrix $\Pi_{\mathcal{W}}^{\bar{C}}$.

Proof. Given $\lambda, \mu$ in $P_{+}$we have

$$
\Pi_{\mathcal{W}}^{\bar{C}}(\mu, \lambda)=K_{\delta, \lambda-\mu} p_{\lambda-\mu} \text { and } \Pi_{\mathcal{H}}(\mu, \lambda)=\frac{m_{\mu, \delta}^{\lambda} s_{\lambda}(x)}{s_{\delta}(x) s_{\mu}(x)} .
$$

If we assume $\delta$ is minuscule, we have $K_{\delta, \lambda-\mu}=m_{\mu, \delta}^{\lambda} \in\{0,1\}$ for any $\lambda, \mu \in P_{+}$by the Remark in $\S 2.3$. Therefore

$$
\Pi_{\mathcal{H}}(\mu, \lambda)=\frac{\psi(\lambda)}{\psi(\mu)} \Pi_{\mathcal{W}}^{\bar{C}}(\mu, \lambda) .
$$

Conversely, if $\Pi_{\mathcal{H}}$ can be realized as a $h$-transform of the substochastic matrix $\Pi_{\mathcal{W}}^{\bar{C}}$ we must have

$$
\frac{h(\lambda)}{h(\mu)} K_{\delta, \lambda-\mu} \frac{x^{\lambda}}{x^{\mu} s_{\delta}(x)}=\frac{m_{\mu, \delta}^{\lambda} s_{\lambda}(x)}{s_{\delta}(x) s_{\mu}(x)} \text { for any } \lambda, \mu \in P_{+} .
$$

This is equivalent to the equality

$$
K_{\delta, \lambda-\mu} \frac{h(\lambda)}{h(\mu)}=m_{\mu, \delta}^{\lambda} \frac{x^{-\lambda} s_{\lambda}(x)}{x^{-\mu} s_{\mu}(x)} \text { for any } \lambda, \mu \in P_{+} .
$$

Since $h$ and $\psi$ are positive functions, there exists a positive function $g$ such that $h(\lambda)=g(\lambda) \psi(\lambda)$, for any $\lambda \in P_{+}$. We thus obtain

$$
K_{\delta, \lambda-\mu} \frac{g(\lambda)}{g(\mu)}=m_{\mu, \delta}^{\lambda} \text { for any } \lambda, \mu \in P_{+} .
$$


Assume (28) holds and $\delta$ is not minuscule. By the Remark in $\S 2.3$, there exists a dominant weight $\kappa \in P_{+}$distinct of $\delta$ such that $K_{\delta, \kappa} \neq 0$. For $\mu=0$ and $\lambda=\kappa$, we then obtain

$$
K_{\delta, \kappa} \frac{g(\kappa)}{g(0)}=m_{0, \delta}^{\kappa}
$$

Now, since $\kappa \neq \delta$, we have $m_{0, \delta}^{\kappa}=0$. Recall that $K_{\delta, \kappa} \neq 0$. This gives $g(\kappa)=0$. Contradiction.

\section{2 $\quad$ Limit of $\psi$ along a drift}

The purpose of the remaining paragraphs of this section is to connect the Markov chain $\mathcal{H}$ to the random walk $\mathcal{W}$ conditioned to never exit $\bar{C}$. We have seen in $\S 5.2$ that, for the minuscule representation $V(\delta)$, the vertices of $B(\delta)^{\otimes \ell}$ can be identified with the paths $Z(\delta, \ell)$. Moreover, by Proposition 5.2.1, the highest weight vertices of $B(\delta)^{\otimes \ell}$ are identified with the paths $Z^{+}(\delta, \ell)$ which remains in $\bar{C}$. The drift $m$ of $\mathcal{W}$ given by Formula (24) belongs to $C$ (the open Weyl chamber) when

$$
m=\sum_{i \in I} m_{i} \omega_{i} \text { with } m_{i}>0 \text { for any } i \in I \text {. }
$$

Lemma 7.2.1 1. We have $m \in C$ if and only if $0<t_{i}<1$ for any $i \in I$.

2. For any direction $\vec{d}$ in $C$, there exists an $n$-tuple $t=\left(t_{1}, \ldots, t_{n}\right)$ with $0<t_{i}<1$ such that $\vec{d}$ is the direction of the drift $m$ associated to the random walk $\mathcal{W}$ defined from $t$ as in $\S$ 5.2.

Proof. 1: Recall that $m=\sum_{b \in B(\delta)} p_{b} \mathrm{wt}(b)$. By (14), for any $b \in B(\delta)$, the coordinates of the weight $\mathrm{wt}(b)$ are determined by the $i$-chains containing $b$. Here by such an $i$-chain, we mean the sub-crystal containing all the vertices connected to $b$ by arrows $i$. By (14) and (22), the contribution of any $i$-chain of length $k$

$$
a_{1} \stackrel{i}{\rightarrow} a_{2} \stackrel{i}{\rightarrow} \cdots \stackrel{i}{\rightarrow} a_{k+1}
$$

to $m$ is equal to

$$
p_{a_{1}} \sum_{j=0}^{[k / 2]}(k-2 j)\left(t_{i}^{j}-t_{i}^{k-j}\right) \omega_{i} .
$$

For each fixed $i$, all these contributions are positive if $0<t_{i}<1$ and they are all nonpositive if $i>1$. This proves Assertion 1 .

2: For any $i=1, \ldots, n$, let $S_{i}$ be the set of vertices $a$ in $B(\delta)$ such that $\varepsilon_{i}(a)=0$. For any $a \in S_{i}$, write $k_{a}$ the length of the $i$-chain in $B(\delta)$ starting at $a$. We denote by $m(t)=\sum_{i=1}^{n} m_{i}(t) \omega_{i} \in C$ the drift corresponding to the $n$-tuple $t=\left(t_{1}, \ldots, t_{n}\right)$ with $0<t_{i}<1$. By the previous arguments

$$
m_{i}(t)=m_{i}\left(t_{i}\right)=\sum_{a \in S_{i}} p_{a} \sum_{j=0}^{\left[k_{a} / 2\right]}\left(k_{a}-2 j\right)\left(t_{i}^{j}-t_{i}^{k_{a}-j}\right)
$$

depends only on $t_{i}$. Write $M_{i}=\max \left\{m_{i}\left(t_{i}\right) \mid 0<t_{i}<1\right\}$. Then $M_{i}>0$ and for any $u_{i}$ in $] 0, M_{i}$ ], there exists $\left.t_{i} \in\right] 0,1\left[\right.$ such that $m_{i}\left(t_{i}\right)=u_{i}$. Now consider a direction $\vec{d}$ in $C$. Assume $v=\left(v_{1}, \ldots, v_{n}\right)$ belongs to $\mathbb{R} \vec{d}$. There exists $c \in \mathbb{R}_{>0}$ such that $\left.\left.u_{i}=c v_{i} \in\right] 0, M_{i}\right]$ for any $i=1, \ldots, n$. It then suffices to choose each $t_{i}$ so that $m_{i}\left(t_{i}\right)=u_{i}$. 
In the sequel, we assume that $m \in C$. Consider a positive root $\alpha$, decomposed as $\alpha=\alpha_{i_{1}}+$ $\cdots+\alpha_{i_{r}}$ on the basis of simple roots. Then $t^{[\alpha]}=t_{i_{1}} \cdots t_{i_{r}}$. According to the fact that $m \in C$, we immediately derive from the previous lemma that $0<t^{[\alpha]}<1$. In particular the product

$$
\nabla=\prod_{\alpha \in R_{+}} \frac{1}{1-x^{-\alpha}}=\prod_{\alpha \in R_{+}} \frac{1}{1-t^{[\alpha]}}
$$

running on the (finite) set of positive roots is well-defined and finite.

Proposition 7.2.2 Assume $m \in C$ and consider a sequence $\left(\lambda^{(a)}\right)_{a \in \mathbb{N}}$ of dominant weights such that $\lambda^{(a)}=a m+o(a)$. Then $\lim _{a \rightarrow+\infty} x^{-\lambda^{(a)}} s_{\lambda^{(a)}}(x)=\nabla$.

Proof. By the Weyl character formula, we have

$$
s_{\lambda^{(a)}}(x)=\nabla \sum_{w \in W} \varepsilon(w) x^{w\left(\lambda^{(a)}+\rho\right)-\rho} .
$$

This gives

$$
x^{-\lambda^{(a)}} s_{\lambda^{(a)}}(x)=\nabla \sum_{w \in W} \varepsilon(w) x^{w\left(\lambda^{(a)}+\rho\right)-\lambda^{(a)}-\rho}=\nabla \sum_{w \in W} \varepsilon(w) t^{\left[\lambda^{(a)}+\rho-w\left(\lambda^{(a)}+\rho\right)\right]} .
$$

For $w=1$, one gets $\varepsilon(w) t^{\left[\lambda^{(a)}+\rho-w\left(\lambda^{(a)}+\rho\right)\right]}=1$. So it suffices to prove that $\lim _{a \rightarrow+\infty} t^{\left[\lambda^{(a)}+\rho-w\left(\lambda^{(a)}+\rho\right)\right]}=$ 0 for any $w \neq 1$. Consider $w \neq 1$ and set

$$
u(a)=\lambda^{(a)}+\rho-w\left(\lambda^{(a)}+\rho\right)=\lambda^{(a)}-w\left(\lambda^{(a)}\right)+\rho-w(\rho) .
$$

Since $m \in C$, the weight $\lambda^{(a)}$ belongs to $C$ for $a$ large enough. In the sequel, we can thus assume that $\lambda^{(a)}+\rho \in C$. Its stabilizer under the action of the Weyl group is then trivial. Now the weights of the finite-dimensional representation $V\left(\lambda^{(a)}+\rho\right)$ are stable under the action of $W$. Thus $w\left(\lambda^{(a)}+\rho\right)$ is a weight of $V\left(\lambda^{(a)}+\rho\right)$. This implies that $w\left(\lambda^{(a)}+\rho\right) \leq \lambda^{(a)}+\rho$, that is $u(a) \in Q_{+}$ is a linear combination of simple root with nonnegative coefficients. Since $\lambda^{(a)}=a m+o(a)$ and $\rho$ is fixed, we have

$$
u(a)=a(m-w(m))+o(a) \in Q_{+} .
$$

For any $w \neq 1$, one gets $m \neq w(m)$. We can set $m-w(m)=\sum_{i=1}^{n} m_{i} \alpha_{i}$ where the $m_{i}$ belong to $\mathbb{R}_{\geq 0}$ for any $i=1, \ldots, n$ and $m_{i_{0}}=\max _{i=1, \ldots, n}\left\{m_{i}\right\}>0$. This gives $u(a)=a \sum_{i=1}^{n} m_{i} \alpha_{i}+o(a)$ and

$$
t^{\left[\lambda^{(a)}+\rho-w\left(\lambda^{(a)}+\rho\right)\right]}=\left(t^{[m]}\right)^{a} \times t^{[o(a)]} \leq t_{i_{0}}^{a m_{i_{0}}} \times t^{[o(a)]}
$$

tends to 0 when $a$ tends to infinity for $0<t_{i_{a}}<1$.

\subsection{Random walks $\mathcal{W}$ with fixed drift}

Let $\delta$ be a minuscule representation. In that case, there is a bijection between the paths from 0 to $\lambda \in P_{+}$and the highest weight vertices of $B(\delta)^{\otimes \ell}$ of weight $\lambda$. Assume the probability distribution on $B(\delta)$ is such that $m \in C$. The following proposition shows that the probability distribution on $B(\delta)$ is completely determined by $m$ under the previous hypotheses. This remark will not be needed in the sequel of the article. 
Proposition 7.3.1 Assume $\delta$ is minuscule. If two probability distributions on $B(\delta)$ satisfying the conditions imposed in $\S 5.3$ have the same drift $m \in C$, then they coincide.

Proof. Assume we have a probability distribution on $B(\delta)$ as in $\S 5.3$. Consider $\lambda^{(\ell)}=\ell m+O(1)$ a sequence of dominants weights which tends to infinity in the direction of the drift $m$. Let $\gamma \in P$. Since $\delta$ is minuscule, all the paths from 0 to $\lambda^{(\ell)}-\gamma\left(\right.$ resp. to $\left.\lambda^{(\ell)}\right)$ have the same probability ; furthermore, there exist $f_{\lambda^{(\ell)}-\gamma, \delta}^{\ell}\left(\right.$ resp. $f_{\lambda^{(\ell)}, \delta}^{\ell}$ ) such paths by Proposition 5.2.1. We thus have

$$
\frac{f_{\lambda^{(\ell)}-\gamma, \delta}^{\ell} x^{\lambda^{(\ell)}-\gamma}}{f_{\lambda^{(\ell)}, \delta}^{\ell} x^{\lambda^{(\ell)}}}=\frac{\mathbb{P}\left(\mathcal{W}_{1} \in \bar{C}, \ldots, \mathcal{W}_{\ell} \in \bar{C}, \mathcal{W}_{\ell}=\lambda^{(\ell)}-\gamma\right)}{\mathbb{P}\left(\mathcal{W}_{1} \in \bar{C}, \ldots, \mathcal{W}_{\ell} \in \bar{C}, \mathcal{W}_{\ell}=\lambda^{(\ell)}\right)}
$$

By Theorem 4.3.1, we know that this quotient tends to 1 when $\ell$ tends to infinity. This gives

$$
\lim _{\ell \rightarrow+\infty} \frac{f_{\lambda^{(\ell)}-\gamma, \delta}^{\ell}}{f_{\lambda^{(\ell)}, \delta}^{\ell}}=x^{\gamma}
$$

hence $x^{\gamma}$ is determined by $m$. Thus the unique probability distribution with drift $m$ defined on $B(\delta)$ verifies $p_{a}=\frac{x^{\mathrm{wt}(a)}}{s_{\delta}(x)}$ where $x^{\mathrm{wt}(a)}$ and $s_{\delta}(x)$ are given by (31).

\subsection{Transition matrix of $\mathcal{W}$ conditioned to never exit the Weyl chamber}

In this paragraph, we assume $\delta$ is minuscule and $m \in C$. Set $\Pi=\Pi_{\mathcal{W}}^{\bar{C}}$. Let us denote by $\Gamma$ the Green function associated to the substochastic matrix $\Pi$. Consider $\mu \in P_{+}$. For any $\lambda \in P$, we have $\Gamma(\mu, \lambda)=\sum_{\ell \geq 0} \Pi^{\ell}(\mu, \lambda)$. Clearly, $\Gamma(\mu, \lambda)=0$ if $\lambda \notin P_{+}$. We consider the Martin Kernel

$$
K\left(\mu, \mathcal{W}_{\ell}\right)=\frac{\Gamma\left(\mu, \mathcal{W}_{\ell}\right)}{\Gamma\left(0, \mathcal{W}_{\ell}\right)}
$$

defined almost surely for $\ell$ large enough.

In order to apply Theorem 3.3.1, we want to prove that $K$ converges almost surely to the harmonic function $\psi$ of $\S 7.1$.

Write $b_{\mu}$ for the highest weight vertex of $B(\mu)$. For $\lambda \in P_{+}$, let $B(\mu, \ell, \lambda)$ be the subset of vertices $b=a_{1} \otimes \cdots \otimes a_{\ell}$ in $B(\delta)^{\otimes \ell}$ such that

$$
\operatorname{wt}\left(b_{\mu} \otimes a_{1} \otimes \cdots \otimes a_{k}\right) \in P_{+} \text {for any } k=1, \ldots, \ell \text { and } \operatorname{wt}\left(b_{\mu} \otimes b\right)=\lambda .
$$

By definition of $\Pi=\Pi_{\mathcal{W}}^{\bar{C}}$, and since $\delta$ is minuscule, we have

$$
\Pi^{\ell}(\mu, \lambda)=\operatorname{card}(B(\mu, \ell, \lambda)) \frac{x^{\lambda-\mu}}{s_{\delta}(x)^{\ell}} .
$$

Indeed, all the paths from $\mu$ to $\lambda$ of length $\ell$ have the same probability $\frac{x^{\lambda-\mu}}{s_{\delta}(x)^{\ell}}$. By (2), Theorem 5.1 .1 and Proposition 5.2.1, we know that

$$
\operatorname{card}(B(\mu, \ell, \lambda))=f_{\lambda / \mu}^{\ell}
$$

and $f_{\lambda / \mu}^{\ell}$ is the number of highest weight vertices of weight $\lambda$ in $B(\mu) \otimes B(\delta)^{\otimes \ell}$. According to Lemma 5.1.3, they can be written as $b_{\mu} \otimes b$ with $\varepsilon_{i}(b) \leq \varphi_{i}\left(b_{\mu}\right)$ for any $i \in I$. 
Remark: When $\delta$ is not minuscule, the vertices $b_{\mu} \otimes b$ with $b \in B(\lambda, \mu, \ell)$ are not necessarily of highest weight and we can have $\operatorname{card}(B(\lambda, \mu, \ell))>f_{\lambda / \mu}^{\ell}$. A vertex of $B(\delta)^{\ell}$ which is not of highest weight can yield a path in $\bar{C}$.

According to the Proposition 5.1.4, given any sequence $\lambda^{(a)}$ of weights of the form $\lambda^{(a)}=$ $a m+o(a)$, we can write for $a$ large enough

$$
\Gamma\left(\mu, \lambda^{(a)}\right)=\sum_{\ell \geq 0} f_{\lambda^{(a)}}^{\ell} \frac{x^{\lambda^{(a)}}-\mu}{s_{\delta}(x)^{\ell}}=x^{\lambda^{(a)}}-\mu \sum_{\ell \geq 0} \frac{1}{s_{\delta}(x)^{\ell}} \sum_{\gamma \in P} f_{\lambda^{(a)}-\gamma}^{\ell} K_{\mu, \gamma}=x^{\lambda^{(a)}}-\mu \sum_{\gamma \in P} K_{\mu, \gamma} \sum_{\ell \geq 0} \frac{f_{\lambda^{\ell(a)}-\gamma}}{s_{\delta}(x)^{\ell}} .
$$

Since

$$
\Gamma\left(0, \lambda^{(a)}\right)=x^{\lambda^{(a)}} \sum_{\gamma \in P} K_{0, \gamma} \sum_{\ell \geq 0} \frac{f_{\lambda^{(a)}-\gamma}^{\ell}}{s_{\delta}(x)^{\ell}}=\sum_{\ell \geq 0} f_{\lambda}^{\ell} \frac{x^{\lambda^{(a)}}}{s_{\delta}(x)^{\ell}},
$$

this yields for $a$ large enough

$$
K\left(\mu, \lambda^{(a)}\right)=x^{-\mu} \sum_{\gamma \in P} K_{\mu, \gamma} \frac{\sum_{\ell \geq 0} \frac{f_{\lambda_{\delta}^{\ell}(a)}^{\ell}(x)^{\ell}}{s_{\delta}}}{\sum_{\ell \geq 0} \frac{f_{\lambda}^{\ell}(a)}{s_{\delta}(x)^{\ell}}}=x^{-\mu} \sum_{\gamma \in P} K_{\mu, \gamma} x^{\gamma} \frac{\sum_{\ell \geq 0} f_{\lambda^{(a)}-\gamma}^{\ell} \frac{x^{\lambda^{(a)}}-\gamma}{s_{\delta}(x)^{\ell}}}{\sum_{\ell \geq 0} f_{\lambda^{(a)}}^{\ell} \frac{x^{\lambda^{(}(a)}}{s_{\delta}(x)^{\ell}}} .
$$

Thus

$$
K\left(\mu, \lambda^{(a)}\right)=x^{-\mu} \sum_{\gamma \text { weight of } V(\mu)} K_{\mu, \gamma} x^{\gamma} \frac{\Gamma\left(0, \lambda^{(a)}-\gamma\right)}{\Gamma\left(0, \lambda^{(a)}\right)} .
$$

Now we have the following proposition

Proposition 7.4.1 Consider $\gamma \in P$ a fixed weight. Then, under the previous assumptions on $m$

$$
\lim _{a \rightarrow+\infty} \frac{\Gamma\left(0, \mathcal{W}_{a}-\gamma\right)}{\Gamma\left(0, \mathcal{W}_{a}\right)}=1 \text { (a.s). }
$$

Proof. The weights $\gamma$ run over the set of weights of $V(\mu)$. This set is finite therefore the statement follows immediately by applying Theorem 4.5.1.

The strong law of large numbers states that $\mathcal{W}_{a}=a m+o(a)$ almost surely. With (33) this implies that almost surely, for $a$ large enough

$$
K\left(\mu, \mathcal{W}_{a}\right)=x^{-\mu} \sum_{\gamma \text { weight of } V(\mu)} K_{\mu, \gamma} x^{\gamma} \frac{\Gamma\left(0, \mathcal{W}_{a}-\gamma\right)}{\Gamma\left(0, \mathcal{W}_{a}\right)} .
$$

Proposition 7.4 .1 then gives

$$
L=\lim _{a \rightarrow+\infty} K\left(\mu, \mathcal{W}_{a}\right)=x^{-\mu} \sum_{\gamma \text { weight of } V(\mu)} K_{\mu, \gamma} x^{\gamma}=x^{-\mu} s_{\mu}(x)=\psi(\mu) \text { (a.s). }
$$

that is, $L$ coincides with the harmonic function of Proposition 7.1.1. By Theorem 3.3.1, there exists a constant $c$ such that $\psi=c h_{\bar{C}}$ where $h_{\bar{C}}$ is the harmonic function defined in $\S 3.2$ associated to the restriction of $\left(\mathcal{W}_{\ell}\right)_{\ell \geq 0}$ to the close Weyl chamber $\bar{C}$. By Theorems 7.1.1 and 6.1.2, we thus derive

$$
\Pi_{h_{\bar{C}}}(\mu, \lambda)=\Pi_{\psi}(\mu, \lambda)=\Pi_{\mathcal{H}}(\mu, \lambda)=\frac{m_{\mu, \delta}^{\lambda} s_{\lambda}(x)}{s_{\delta}(x) s_{\mu}(x)}=\frac{s_{\lambda}(x)}{s_{\delta}(x) s_{\mu}(x)}
$$


since $\delta$ is a minuscule representation (so that $m_{\mu, \delta}^{\lambda}=1$ for any $\lambda, \mu \in P_{+}$such that $\lambda-\mu$ is a weight of $B(\delta)($ see $\S 2.3))$.

Theorem 7.4.2 Assume $\delta$ is a minuscule representation and $m \in C$. Then the transition matrix of the Markov chain $\mathcal{H}$ is the same as the transition matrix of $\left(\mathcal{W}_{\ell}^{\bar{C}}\right)_{\ell \geq 0}$, which is the random walk $\mathcal{W}$ conditioned to never exit the cone $\bar{C}$. That is, the corresponding transition probabilities are given by

$$
\Pi_{\mathcal{H}}(\mu, \lambda)=\frac{s_{\lambda}(x)}{s_{\delta}(x) s_{\mu}(x)} \text { for any } \lambda, \mu \in P_{+} \text {such that } \lambda-\mu \text { is a weight of } B(\delta) .
$$

Corollary 7.4.3 With the above notation and assumptions we have for any $\lambda \in P_{+}$

$$
\mathbb{P}_{\lambda}\left(\mathcal{W}_{\ell} \in \bar{C} \text { for any } \ell \geq 1\right)=x^{-\lambda} s_{\lambda}(x) \prod_{\alpha \in R_{+}}\left(1-x^{-\alpha}\right) .
$$

Proof. Recall that the function $\lambda \longmapsto \mathbb{P}_{\lambda}\left(\mathcal{W}_{\ell} \in \bar{C}\right.$ for any $\left.\ell \geq 1\right)$ is harmonic. By Theorem 7.4.2, there is a positive constant $c$ such that $\mathbb{P}_{\lambda}\left(\mathcal{W}_{\ell} \in \bar{C}\right.$ for any $\left.\ell \geq 1\right)=c x^{-\lambda} s_{\lambda}(x)$. Now, for any sequence $\lambda^{(a)}=a m+o(a)$ of dominant weights

$$
\lim _{a \rightarrow+\infty} \mathbb{P}_{\lambda^{(a)}}\left(\mathcal{W}_{\ell} \in \bar{C} \text { for any } \ell \geq 1\right)=\lim _{a \rightarrow+\infty} \mathbb{P}_{0}\left(\mathcal{W}_{\ell}+\lambda^{(a)} \in \bar{C} \text { for any } \ell \geq 1\right)=1
$$

On the other hand, we know by Proposition 7.2.2 that $\lim _{a \rightarrow+\infty} x^{-\lambda} s_{\lambda}(x)=\nabla$ (see (30) $)$. Therefore $c=\frac{1}{\nabla}$ and we are done.

Examples 7.4.4 Consider the random walk $\mathcal{W}_{\ell}$ in $\mathbb{Z}^{n}$, with steps from one point to one of the four nearest neighbors, and the condition $p_{a_{i}} p_{a_{\bar{i}}}$ independent of $i$ (see Example 5.3.1). Let $\nu \in P_{+}$. Let us compute $\mathbb{P}_{\nu}\left(\mathcal{W}_{\ell} \in \bar{C}\right.$ for any $\left.\ell \geq 1\right)$ for

$$
\begin{aligned}
& \bar{C}=\left\{\lambda \mid \lambda_{1} \geq \cdots \geq \lambda_{n-1} \geq \lambda_{n} \geq 0, \lambda_{i} \in \mathbb{Z}\right\} \text { and } \\
& \bar{C}=\left\{\lambda\left|\lambda_{1} \geq \cdots \geq \lambda_{n-1} \geq\right| \lambda_{n} \mid \geq 0, \lambda_{i} \in \mathbb{Z} \cup \frac{1}{2} \mathbb{Z}\right\}
\end{aligned}
$$

that is for the Weyl chambers of types $C_{n}$ and $D_{n}$.

1. In type $C_{n}$, the process $\mathcal{W}_{\ell}$ is obtained from $B\left(\omega_{1}\right)$. The simple roots are the $\alpha_{i}=\varepsilon_{i}-\varepsilon_{i+1}, i=$ $1, \ldots, n-1$ and $\alpha_{n}=2 \varepsilon_{n}$, we obtain $x_{i}=\frac{1}{t_{i} \cdots t_{n-1} \sqrt{t_{n}}}$ for any $i=1, \ldots n-1$ and $x_{n}=\frac{1}{\sqrt{t_{n}}}$. The positive roots are $\varepsilon_{i} \pm \varepsilon_{i}$ with $1 \leq i<j \leq n$ and $2 \varepsilon_{i}$ with $i=1, \ldots, n$. The desired probability is therefore

$$
\mathbb{P}_{\nu}\left(\mathcal{W}_{\ell} \in \bar{C} \text { for any } \ell \geq 1\right)=x^{-\nu} s_{\nu}^{C_{n}}(x) \prod_{1 \leq i<j \leq n}\left(1-\frac{x_{j}}{x_{i}}\right)\left(1-\frac{1}{x_{i} x_{j}}\right) \prod_{1 \leq i \leq n}\left(1-\frac{1}{x_{i}^{2}}\right)
$$

where $s_{\nu}^{C_{n}}(x)$ is the Weyl character of type $C_{n}$ associated to $\nu$ specialized in $x_{1}, \ldots, x_{n}$.

2. In type $D_{n}$, the process $\mathcal{W}_{\ell}$ is also obtained from $B\left(\omega_{1}\right)$. The simple roots are the $\alpha_{i}=$ $\varepsilon_{i}-\varepsilon_{i+1}, i=1, \ldots, n-1$ and $\alpha_{n}=\varepsilon_{n-1}+\varepsilon_{n}$. We obtain $x_{i}=\frac{1}{t_{i} \cdots t_{n-2} \sqrt{t_{n-1} t_{n}}}$ for any $i=1, \ldots, n-2, x_{n-1}=\frac{1}{\sqrt{t_{n-1} t_{n}}}$ and $x_{n}=\sqrt{\frac{t_{n-1}}{t_{n}}}$. The positive roots are $\varepsilon_{i} \pm \varepsilon_{i}$ with $1 \leq i<j \leq n$.

$$
\mathbb{P}_{\nu}\left(\mathcal{W}_{\ell} \in \bar{C} \text { for any } \ell \geq 1\right)=x^{-\nu} s_{\nu}^{D_{n}}(x) \prod_{1 \leq i<j \leq n}\left(1-\frac{x_{j}}{x_{i}}\right)\left(1-\frac{1}{x_{i} x_{j}}\right)
$$

where $s_{\nu}^{D_{n}}(x)$ is the Weyl character of type $D_{n}$ associated to $\nu$ specialized in $x_{1}, \ldots, x_{n}$. 


\section{Complementary results}

\subsection{Asymptotic behavior of the coefficients $f_{\lambda / \mu, \delta}^{\ell}$}

In 23], Stanley studied the asymptotic behavior of $f_{\lambda / \mu, \delta}^{\ell}$ when $V(\delta)$ is the defining representation of $\mathfrak{g l}_{n}$ (i.e. $\delta=\omega_{1}$ is associated to the classical ballot problem). More precisely, he established that for any fixed $\mu \in P_{+}$and any direction $\vec{d}$ in $C$

$$
\lim _{\ell \rightarrow \infty} \frac{f_{\lambda^{(\ell) / \mu, \omega_{1}}}^{\ell}}{f_{\lambda^{(\ell)}, \omega_{1}}^{\ell}}=s_{\mu}(m)
$$

where $m=\left(m_{1}, \ldots, m_{n}\right) \in d$ is such that $m_{i} \geq 0$ for any $i=1, \ldots n$, the sum $m_{1}+\cdots+m_{n}$ equals 1 and $\lambda^{(\ell)}=\ell m+o(\ell)$ tends to $\infty$ in the direction $\vec{d}$.

By the previous theorem, one may extend this result as follows. Assume $\vec{d}$ is a direction in $C$. By 2 of Lemma 7.2.1, there exists a $n$-tuple $t=\left(t_{1}, \ldots, t_{n}\right)$ with $0<t_{i}<1$ such that $\vec{d}$ is the direction of the drift $m \in C$ associated to the random walk $\mathcal{W}$ defined from $t$ as in $\S 5.2$.

Theorem 8.1.1 Assume $\delta$ is minuscule. If $\lambda^{(\ell)}=\ell m+o\left(\ell^{\alpha}\right)$ with $\alpha<2 / 3$, then

$$
\lim _{\ell \rightarrow \infty} \frac{f_{\lambda^{(\ell) / \mu, \delta}}^{\ell}}{f_{\lambda^{(\ell)}, \delta}^{\ell}}=s_{\mu}(x)
$$

Proof. Consider $\lambda^{(\ell)}=\ell m+o(\ell)$ a sequence of dominants weights which tends to infinity in the direction $\vec{d}$. By Proposition 5.1.4

$$
\frac{f_{\lambda^{(\ell)} / \mu, \delta}^{\ell}}{f_{\lambda^{(\ell)}, \delta}^{\ell}}=\sum_{\gamma \in P} K_{\mu, \gamma} \frac{f_{\lambda^{(\ell)}-\gamma, \delta}^{\ell}}{f_{\lambda^{(\ell)}, \delta}^{\ell}}=\sum_{\gamma \in P} K_{\mu, \gamma} \frac{f_{\lambda^{(\ell)}-\gamma, \delta}^{\ell} x^{\lambda^{(\ell)}}-\gamma}{f_{\lambda^{(\ell)}, \delta}^{\ell} x^{\lambda^{(\ell)}}} x^{\gamma}
$$

where the sums are finite since the set of weight in $V(\mu)$ is finite. Note that, for any $\gamma \in P$

$$
\frac{f_{\lambda^{(\ell)}-\gamma, \delta^{\ell}}^{x^{\lambda^{(\ell)}}-\gamma}}{f_{\lambda^{(\ell)}, \delta}^{x^{\lambda^{(\ell)}}}}=\frac{\mathbb{P}\left(\mathcal{W}_{1} \in \bar{C}, \ldots, \mathcal{W}_{\ell} \in \bar{C}, \mathcal{W}_{\ell}=\lambda^{(\ell)}-\gamma\right)}{\mathbb{P}\left(\mathcal{W}_{1} \in \bar{C}, \ldots, \mathcal{W}_{\ell} \in \bar{C}, \mathcal{W}_{\ell}=\lambda^{(\ell)}\right)}
$$

By Theorem 4.3.1, we know that this quotient tends to 1 when $\ell$ tends to infinity. This implies

$$
\lim _{\ell \rightarrow+\infty} \frac{f_{\lambda^{(\ell)} / \mu, \delta}^{\ell}}{f_{\lambda^{(\ell)}, \delta}^{\ell}}=\sum_{\gamma \in P} K_{\mu, \gamma} x^{\gamma}=s_{\mu}(x)
$$

as announced.

Remark: When $\delta=\omega_{1}$ in type $A_{n-1}$ (ballot problem), we have $p_{i}=\frac{x_{i}}{s_{\delta}(x)}$. Then $m=\frac{1}{s_{\delta}(x)} \sum_{i=1}^{n} x_{i} \varepsilon_{i}$. We can normalize the law so that $s_{\delta}(x)=1$ (it suffices to replace each $x_{i}$ by $\frac{x_{i}}{s_{\delta}(x)}$ ). Then $p_{i}=m_{i}=x_{i}$. This shows that our theorem can indeed be regarded as a generalization of (35)). Finally note that the proof of (35) mainly uses the representation theory of the symmetric group. It seems nevertheless difficult to obtain a purely algebraic proof of the limits (36). 


\subsection{Random walks defined from non irreducible representations}

In Section 6, we have defined the random walk $\left(\mathcal{W}_{\ell}\right)_{\ell \geq 0}$ starting from the crystal $B(\delta)$ of the irreducible module $V_{q}(\delta)$. In fact, most of our results can be easily adapted to the case where $\left(\mathcal{W}_{\ell}\right)_{\ell \geq 0}$ is defined from the crystal $B(M)$ of a $U_{q}(\mathfrak{g})$-module $M$ possibly non irreducible. These random walks are therefore based on tensor products of non irreducible representations. Such products will also provide us with random walks for which the conditioned law to never exit $\bar{C}$ can be made explicit in terms of characters.

To do this, we consider similarly $t_{1}, \ldots, t_{n}$ some positive real numbers and $x_{1}, \ldots, x_{N}$ such that $x^{\alpha_{i}}=t_{i}^{-1}$. This permits to define a probability distribution on $B(M)$, setting $p_{a}=\frac{x^{\mathrm{wt}(a)}}{s_{M}(x)}$ where $s_{M}$ is the character of $M$, that is the sum of the characters of its irreducible components. The random variable $X$ on $B(M)$ is such that $X(a)=\operatorname{wt}(a)$ for any $a \in B(M)$. The product probability distribution on $B(M)^{\otimes \ell}$ verifies $p_{b}=\frac{x^{\mathrm{wt}(b)}}{s_{M}(x)^{\ell}}$ for any $b \in B(\delta)^{\otimes \ell}$. We introduce similarly $\Omega(M)$ as the projective limit of the tensor products $B(M)^{\otimes \ell}$ and the Markov chain $\mathcal{W}=\left(\mathcal{W}_{\ell}\right)_{\ell \geq 0}$ such that $\mathcal{W}_{\ell}(b)=\operatorname{wt}\left(b^{(\ell)}\right)$ for any $\ell \geq 0$. Its transition matrix verifies

$$
\Pi_{\mathcal{W}}\left(\beta, \beta^{\prime}\right)=K_{M, \beta^{\prime}-\beta} \frac{x^{\beta^{\prime}-\beta}}{s_{M}(x)}
$$

where $K_{M, \beta^{\prime}-\beta}$ is the dimension of the weight space $\beta^{\prime}-\beta$ in $M$. Write $M=\oplus_{\nu \in P_{+}} V_{q}^{\oplus m_{\nu}}(\nu)$ for the decomposition of $M$ into its irreducible components. Then the set of increments for the Markov chain corresponding to $M$ is the union of the sets of transition for the Markov chains corresponding to each $V(\nu)$ with $m_{\nu}>0$. Observe that the Markov chain obtained for an isotypical representation $M=V_{q}^{\oplus m_{\nu}}(\nu)$ is the same as the Markov chain for $V(\nu)$. Nevertheless when $M$ admits at least two non isomorphic irreducible components, the matrix $\Pi_{\mathcal{W}}$ depends on the multiplicities $m_{\nu}$.

One can consider the random variable $\mathcal{H}_{\ell}$ on $\Omega(M)$ such that $\mathcal{H}_{\ell}(b)=\operatorname{wt}(\mathfrak{P}(b(\ell)))$ for any $b \in \Omega(M)$, that is $\mathcal{H}_{\ell}(b)$ is the highest weight of $B(b)$, the connected component of $B(M)^{\otimes \ell}$ containing $b^{(\ell)}$. In the proof of Theorem 6.1.2, we do not use the fact that $B(\delta)$ is connected (or equivalently that $V_{q}(\delta)$ is irreducible). Then the same proof shows that $\mathcal{H}=\left(\mathcal{H}_{\ell}\right)_{\ell \geq 0}$ is yet a Markov chain with transition probabilities

$$
\Pi_{\mathcal{H}}(\mu, \lambda)=\frac{m_{\mu, M}^{\lambda} s_{\lambda}(x)}{s_{M}(x) s_{\mu}(x)}
$$

where $m_{\mu, M}^{\lambda}$ is the multiplicity of $V_{q}(\lambda)$ in $V_{q}(\mu) \otimes M$.

In order to obtain an analogue of Proposition 7.1.1, we will say that $M$ is of minuscule type if all its irreducible components are minuscule representations and $\operatorname{dim} M_{\mu} \in\{0,1\}$ for any weight $\mu$. In particular, the nonzero multiplicities in the decomposition $M=\oplus_{\nu \in P_{+}} V_{q}(\nu)$ in irreducible are equal to 1 . When $M$ is of minuscule type, we thus have $K_{M, \lambda-\mu}=m_{\mu, M}^{\lambda} \in\{0,1\}$ for any $\lambda, \mu \in P_{+}$ since $K_{\nu, \lambda-\mu}=m_{\mu, \nu}^{\lambda}$ for any $\nu$ and the irreducible components $V_{q}(\nu)$ have no common weight. We give below the table of the possible non irreducible minuscule type representations for each root system. For a table of the minuscule representations see $\S 2.3$.

\begin{tabular}{|l|l|}
\hline type & non irreducible minuscule type representations \\
\hline$A_{n}$ & $\oplus_{j \in J} V\left(\omega_{j}\right)$ for $J \subset\{1, \ldots, n-1\}$ and $|J|>1$ \\
\hline$D_{n}$ & $\oplus_{j \in J} V\left(\omega_{j}\right)$ for $J \subset\{1, n-1, n\}$ and $|J|>1$ \\
\hline$E_{6}$ & $V\left(\omega_{1}\right) \oplus V\left(\omega_{6}\right)$. \\
\hline
\end{tabular}


Theorem 8.2.1 Assume $M$ is of minuscule type. Then, the transition matrix $\Pi_{\mathcal{H}}$ can be realized as a $\psi$-transform of the substochastic matrix $\Pi_{\mathcal{W}}^{\bar{C}}$ where $\psi$ is the harmonic function defined by $\psi(\lambda)=x^{-\lambda} s_{\lambda}(x)$ for any $\lambda \in P_{+}$.

Proof. Write $M=\oplus_{\nu \in P_{+}} V_{q}(\nu)$ the decomposition of $M$ in its irreducible components. We have

$$
K_{M, \lambda-\mu}=\sum_{\nu} K_{\nu, \lambda-\mu} \in\{0,1\} \text { and } m_{\mu, M}^{\lambda}=\sum_{\nu} m_{\mu, \nu}^{\lambda} \in\{0,1\} .
$$

Similarly to the proof of Proposition 7.1.1, the matrix $\Pi_{\mathcal{H}}$ can be realized as the $\psi$-transform of the substochastic matrix $\Pi_{\mathcal{W}}^{\bar{C}}$ since we have $K_{M, \lambda-\mu}=m_{\mu, M}^{\lambda}$ for any $\lambda, \mu \in P_{+}$.

In the rest of this paragraph, we assume that $m=\mathbb{E}(X)$ belongs to $C$. As in Lemma 7.2.1, this is equivalent to the assumption $0<t_{i}<1$ for any $i=1, \ldots, n$. If we denote by $f_{\lambda / \mu, M}^{\ell}$ the multiplicity of $V_{q}(\lambda)$ in $V_{q}(\mu) \otimes M^{\otimes \ell}$, the decomposition of Proposition 5.1.4 yet holds. Moreover, Proposition 5.2 .1 admits a straightforward analogue which guarantees that each vertex $b_{\mu} \otimes b$ with $b \in B(M)^{\otimes \ell}$ yielding a path in $\bar{C}$ is of highest weight. If we consider $\lambda^{(a)}$ a sequence of weights of the form $\lambda^{(a)}=a m+o(a)$, one has for $a$ large enough, $\lambda^{(a)} \in P_{+}$and

$$
f_{\lambda^{(a)} / \mu, M}^{\ell}=\sum_{\kappa \in P_{+}} f_{\kappa, M}^{\ell} K_{\mu, \lambda(a)-\kappa}=\sum_{\gamma \in P} f_{\lambda^{(a)}-\gamma, M}^{\ell} K_{\mu, \gamma}
$$

The proof of Theorem 7.4 .2 leads to the

Theorem 8.2.2 Assume $M$ is a minuscule type representation and $m \in C$. Then the transition matrix of $\left(\mathcal{W}_{\ell}^{\bar{C}}\right)_{\ell \geq 0}$ is the same as the transition matrix of the Markov chain $\mathcal{H}$. That is, the corresponding transition probabilities are given by

$$
\Pi(\mu, \lambda)=\frac{s_{\lambda}(x)}{s_{M}(x) s_{\mu}(x)} \text { for any } \lambda, \mu \in P_{+} \text {such that } \lambda-\mu \text { is a weight of } B(M) \text {. }
$$

Example 8.2.3 Consider the minuscule type representation $M=V\left(\omega_{1}\right) \oplus V\left(\omega_{n-1}\right) \oplus V\left(\omega_{n}\right)$ in type $D_{n}$. The simple roots are the $\alpha_{i}=\varepsilon_{i}-\varepsilon_{i+1}, i=1, \ldots, n-1$ and $\alpha_{n}=\varepsilon_{n-1}+\varepsilon_{n}$. We thus have $t_{i}=\frac{x_{i+1}}{x_{i}}$ for any $i=1, \ldots, n-1$ and $t_{n}=\frac{1}{x_{n-1} x_{n}}$. This gives $x_{i}=\frac{1}{t_{i} \cdots t_{n-2} \sqrt{t_{n-1} t_{n}}}$ for any $i=1, \ldots, n-2, x_{n-1}=\frac{1}{\sqrt{t_{n-1} t_{n}}}$ and $x_{n}=\sqrt{\frac{t_{n-1}}{t_{n}}}$. We have $s_{M}(x)=s_{\omega_{1}}(x)+s_{\omega_{n-1}}(x)+s_{\omega_{n}}(x)$. The weights $\mu$ of $M$ (an thus the possible transitions for $\mathcal{W}$ in $P$ ) are such that

$$
\mu \in\left\{ \pm \varepsilon_{i} \mid i=1, \ldots, n\right\} \sqcup\left\{ \pm \frac{1}{2} \varepsilon_{1} \pm \cdots \pm \frac{1}{2} \varepsilon_{n}\right\} .
$$

We thus have $2^{n}+2 n$ possible transitions and the probability corresponding to the transition $\mu$ is $p_{\mu}=\frac{x^{\mu}}{s_{M}(x)}$.

\section{Appendix: Miscellaneous proofs}

\subsection{Proof of Theorem 3.3.1}

The probability space on which the chain is defined is equipped with the probability $\mathbb{P}_{x^{*}}$ defining the chain issued from the particular point $x^{*}$. 
Denote by $K_{h}$ the Martin kernel associated to $\Pi_{h}$; for any $x, y \in M$ one gets

$$
K_{h}(x, y)=\frac{h\left(x^{*}\right)}{h(x)} K(x, y) .
$$

The Markov chain $\left(Y_{\ell}^{h}\right)$ is transient in $M$ and it converges almost surely in the Martin topology to a random variable $Y_{\infty}^{h}$ taking values in the Martin boundary $\mathcal{M}$ (see for instance Theorem 7.19 in

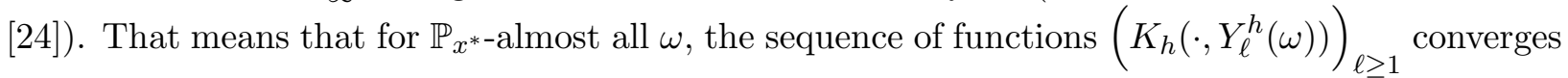
pointwise to some (random) function $K_{h}\left(\cdot, Y_{\infty}^{h}(\omega)\right)$; in this context, the hypothesis of the theorem may be written

$$
\forall x \in M, \quad K_{h}\left(x, Y_{\infty}^{h}(\omega)\right)=\frac{h\left(x^{*}\right)}{h(x)} f(x) \quad \text { a.s. . }
$$

Since the family of functions $\left\{K_{h}(\cdot, \xi), \xi \in \mathcal{M}\right\}$ separates the boundary points, the function $K_{h}\left(\cdot, Y_{\infty}^{h}(\omega)\right)$ is almost surely constant, i-e $Y_{\infty}^{h}(\omega)=\xi_{\infty}$ a.s. for some fixed boundary point $\xi_{\infty}$.

It remains to prove that $K_{h}\left(x, \xi_{\infty}\right)$ does not depend on $x$. Note that $\mathbb{P}_{x} \ll \mathbb{P}_{x^{\star}}$ for all $x \in M$, since $\Gamma\left(x^{*}, x\right)>0$. Setting $\nu_{x}(B):=\mathbb{P}_{x}\left[Y_{\infty}^{h} \in B\right]$ for any Borel set $B$ in $\mathcal{M}$ and any $x \in M$, one gets (see for instance Theorem 7.42 in [24])

$$
\nu_{x}(B)=\int_{B} K_{h}(x, \xi) \nu_{x^{*}}(d \xi)
$$

By the above, $\nu_{x^{*}}$ is the Dirac mass at the point $\xi_{\infty}$; the equality (39) with $B=M$ gives $1=K_{h}\left(x, \xi_{\infty}\right)$ for any $x \in M$, i-e $f(x)=\frac{h(x)}{h\left(x^{*}\right)}$. The proof is complete.

\subsection{Proof of Lemma 4.3 .2}

We begin by a lemma coming from Garbit's thesis ([6], [7]). This lemma gives a uniform lower bound for the probability that a centered random walk goes from the ball of radius $r+1$ to the ball of radius $r$ without leaving a cone.

Let $\left(R_{\ell}\right)_{\ell \geq 1}$ be a centered random walk in $\mathbb{R}^{n}$, with finite second moment. Let $\mathcal{C}_{0}$ be an open convex cone in $\mathbb{R}^{n}$. We fix a unitary vector $\vec{u}$ in $\mathcal{C}_{0}$. For any $a>0$, we denote by $\mathcal{C}_{a}$ the translated cone $\mathcal{C}_{a}:=a \vec{u}+\mathcal{C}_{0}$, and for any $r>0$ we denote by $\mathcal{C}_{a}(r)$ the truncated translated cone $\mathcal{C}_{a}(r):=\left\{x \in \mathcal{C}_{a} \mid\|x\| \leq r\right\}$, which is non-empty if $a<r$.

Lemma 9.2.1 ([6], [7]) There exist a positive integer $k$ and real positive numbers $r_{0}$, a and $\rho$, with $a \leq r_{0}$ such that

$$
\forall r \geq r_{0}, \forall x \in \mathcal{C}_{a}(r+1), \mathbb{P}_{x}\left[R_{1} \in \mathcal{C}_{0}, \ldots, R_{k} \in \mathcal{C}_{0}, R_{k} \in \mathcal{C}_{a}(r)\right] \geq \rho .
$$

Proof of Lemma 9.2.1. We define, for $y \in \mathbb{R}^{n}$ and $\ell \in \mathbb{N}$,

$$
p_{\ell}(y):=\mathbb{P}_{0}\left[y+\frac{R_{1}}{\sqrt{\ell}} \in \mathcal{C}_{0}, \ldots, y+\frac{R_{\ell}}{\sqrt{\ell}} \in \mathcal{C}_{0}, y+\frac{R_{\ell}}{\sqrt{\ell}} \in \mathcal{C}_{1}(2)\right]
$$

We have also

$$
p_{\ell}(y)=\mathbb{P}_{\sqrt{\ell} y}\left[R_{1} \in \mathcal{C}_{0}, \ldots, R_{\ell} \in \mathcal{C}_{0}, R_{\ell} \in \mathcal{C}_{\sqrt{\ell}}(2 \sqrt{\ell})\right]
$$


If a sequence $\left(y_{\ell}\right)$ in $\mathbb{R}^{n}$ converges to a point $y$ in $\mathcal{C}_{0}$, then the "Donsker line" going through the points $\left(y_{\ell}+\frac{R_{i}}{\sqrt{\ell}}\right)_{i=1, \ldots, \ell}$ converges in law to a Brownian path (between times 0 and 1 ) starting at $y$; the probability that this Brownian path stays in $\mathcal{C}_{0}$ and ends in $\mathcal{C}_{1}(2)$ is positive ; from Portemanteau theorem (c.f.[2]), we deduce that $\liminf p_{\ell}\left(y_{\ell}\right)>0$. It follows that, for any compact $K \subset \mathcal{C}_{0}$, there exists a positive integer $\ell$ such that $\inf _{y \in K} p_{\ell}(y)>0$.

We choose a positive integer $k$ and a positive real $\rho$ such that, for all $y \in \overline{\mathcal{C}_{1}(3)}, p_{k}(y) \geq \rho$. We note that if $\sqrt{k} y \in \mathcal{C}_{\sqrt{k}}(2 \sqrt{k}+1)$ then $y \in \overline{\mathcal{C}_{1}(3)}$, and we conclude that

$$
\forall z \in \mathcal{C}_{\sqrt{k}}(2 \sqrt{k}+1), \mathbb{P}_{z}\left[R_{1} \in \mathcal{C}_{0}, \ldots, R_{k} \in \mathcal{C}_{0}, R_{k} \in \mathcal{C}_{\sqrt{k}}(2 \sqrt{k})\right] \geq \rho .
$$

We define $r_{0}:=2 \sqrt{k}$ and $a:=\sqrt{k}$. The four parameters of the lemma are now fixed, and the result is proved for $r=r_{0}$.

We consider now $r>r_{0}$ and $z \in \mathcal{C}_{a}(r+1)$ and we distinguish two cases.

- If $z \in \mathcal{C}_{a}\left(r_{0}+1\right)$ then $\mathbb{P}_{z}\left[R_{1} \in \mathcal{C}_{0}, \ldots, R_{k} \in \mathcal{C}_{0}, R_{k} \in \mathcal{C}_{a}(r)\right] \geq \rho$, because $\mathcal{C}_{a}\left(r_{0}\right) \subset \mathcal{C}_{a}(r)$ and $\mathbb{P}_{z}\left[R_{1} \in \mathcal{C}_{0}, \ldots, R_{k} \in \mathcal{C}_{0}, R_{k} \in \mathcal{C}_{a}\left(r_{0}\right)\right] \geq \rho$.

- If $z \notin \mathcal{C}_{a}\left(r_{0}+1\right)$ we remark that $\|a \vec{u}\|<r_{0}+1<\|z\|$ and we denote by $z^{\prime}$ the point on the segment $[a \vec{u}, z]$ such that $\left\|z^{\prime}\right\|=r_{0}+1$. We have $z^{\prime} \in \mathcal{C}_{a}\left(r_{0}+1\right)$ and we verify, by inclusion of events, that

$$
\mathbb{P}_{z^{\prime}}\left[R_{1} \in \mathcal{C}_{0}, \ldots, R_{k} \in \mathcal{C}_{0}, R_{k} \in \mathcal{C}_{a}\left(r_{0}\right)\right] \leq \mathbb{P}_{z}\left[R_{1} \in \mathcal{C}_{0}, \ldots, R_{k} \in \mathcal{C}_{0}, R_{k} \in \mathcal{C}_{a}(r)\right]
$$

We conclude that this last term is $\geq \rho$.

The proof of the lemma is complete.

Proof of Lemma 4.3.2. We come back now to the study of a sequence of bounded independent identically distributed random variables $\left(X_{\ell}\right)$ taken values in a discrete $\operatorname{subgroup} G$ of $\mathbb{R}^{n}$. We denote by $m$ there common mean and by $\left(S_{\ell}\right)$ the associated random walk. Moreover we consider a cone $\mathcal{C}$ and an open convex subcone $\mathcal{C}_{0}$ satisfying the assumptions stated in Section 4.1 .

Let $\alpha \in] 1 / 2,2 / 3\left[\right.$. We suppose that the sequence $\left(\ell^{-\alpha}\left\|g_{\ell}-\ell m\right\|\right)$ is bounded, and we want to prove that

$$
\liminf \left(\mathbb{P}\left[S_{1} \in \mathcal{C}, \ldots, S_{\ell} \in \mathcal{C}, S_{\ell}=g_{\ell}\right]\right)^{\ell^{-\alpha}}>0 .
$$

It is sufficient to prove this result with $\mathcal{C}$ replaced by $\mathcal{C}_{0}$, so we suppose in the sequel that $\mathcal{C}$ satisfies the assumptions imposed to $\mathcal{C}_{0}$. By Lemma 4.1.1, we know that

$$
p:=\mathbb{P}\left[\forall \ell \geq 1, S_{\ell} \in \mathcal{C}\right]>0 .
$$

Since $\alpha>1 / 2$, the sequence $\ell^{-\alpha}\left(S_{\ell}-\ell m\right)$ goes to zero in quadratic mean, hence in probability. For $r_{0}$ large enough, for all $\ell$, we have

$$
\mathbb{P}\left[S_{1} \in \mathcal{C}, \ldots, S_{\ell} \in \mathcal{C},\left\|S_{\ell}-\ell m\right\| \leq r_{0}+\left[\ell^{\alpha}\right]\right] \geq p / 2 .
$$

From Lemma 9.2.1 applied to the random walk $R_{\ell}=S_{\ell}-\ell m$, we know that there exist an integer $k>0$, a positive real number $r_{0}$ and a positive real number $\rho$ such that, for all $r \geq r_{0}$ and all $x \in \mathcal{C}$ with $\|x\| \leq r+1$,

$$
\mathbb{P}_{x}\left[S_{1}-m \in \mathcal{C}, \ldots, S_{k}-k m \in \mathcal{C},\left\|S_{k}-k m\right\| \leq r\right] \geq \rho
$$


All the more, for all $r>r_{0}$ and all $x \in \mathcal{C}$ with $\|x\| \leq r+1$,

$$
\mathbb{P}_{x}\left[S_{1} \in \mathcal{C}, \ldots, S_{k} \in \mathcal{C},\left\|S_{k}-k m\right\| \leq r\right] \geq \rho .
$$

We fix $k, r_{0}$ and $\rho$.

Looking to a path $\left(S_{i}\right)_{1 \leq i \leq \ell+k\left[\ell^{\alpha}\right]}$ staying in the cone and such that $\left\|S_{\ell}-\ell m\right\| \leq r_{0}+\left[\ell^{\alpha}\right]$ and $\left\|S_{\ell+k j}-(\ell+k j) m\right\| \leq r_{0}+\left[\ell^{\alpha}\right]-j, 1 \leq j \leq\left[\ell^{\alpha}\right]$, we deduce from (40) and (41) that, for all $\ell$,

$$
\mathbb{P}\left[S_{1} \in \mathcal{C}, \ldots, S_{\ell+k\left[\ell^{\alpha}\right]} \in \mathcal{C},\left\|S_{\ell+k\left[\ell^{\alpha}\right]}-\left(\ell+k\left[\ell^{\alpha}\right]\right) m\right\| \leq r_{0}\right] \geq \frac{p}{2} \rho^{\ell^{\alpha}} .
$$

The distance between the ball $B\left(\ell m, r_{0}\right)$ and the complementary of the cone $\mathcal{C}$ increases linearly with $\ell$. We can fix an integer $d$ such that, for all $\ell$, the distance between the ball $B\left(d \ell m, r_{0}\right)$ and the complementary of the cone is greater than $2 \ell$ times the $L^{\infty}$ norm of the random variables $X_{\ell}$.

Let us summarize what we know :

- For all $\ell$,

$$
\mathbb{P}\left[S_{1} \in \mathcal{C}, \ldots, S_{d \ell+k\left[(d \ell)^{\alpha}\right]} \in \mathcal{C},\left\|S_{d \ell+k\left[(d \ell)^{\alpha}\right]}-\left(d \ell+k\left[(d \ell)^{\alpha}\right]\right) m\right\| \leq r_{0}\right] \geq \frac{p}{2} \rho^{(d \ell)^{\alpha}} .
$$

- Starting from a point in the ball $B\left(\left(d \ell+k\left[(d \ell)^{\alpha}\right]\right) m, r_{0}\right)$, the random walk cannot exit the cone $\mathcal{C}$ in less than $2 \ell$ steps.

We want now to apply Theorem 4.2.1; for all integers $\ell, i>0$ with $\ell \leq i \leq 2 \ell$ we define $g_{\ell, i}^{\prime}:=g_{d \ell+k\left[(d \ell)^{\alpha}\right]+i}-x-\left(d \ell+k\left[(d \ell)^{\alpha}\right]\right) m$ and we notice that, if $\|x\| \leq r_{0}$, then

$$
\left\|g_{\ell, i}^{\prime}-i m\right\|=O\left(\left(d \ell+k\left[(d \ell)^{\alpha}\right]+i\right)^{\alpha}\right)=O\left(i^{\alpha}\right) .
$$

Since $\alpha<2 / 3$, Theorem 4.2.1 gives us the existence of positive constants $c$ and $C$ such that, for all $\ell$ large enough,

$$
\inf _{x:\|x\| \leq r_{0} \text { and } g_{\ell, i}^{\prime} \in G} \mathbb{P}_{x}\left[S_{i}=g_{\ell, i}^{\prime}\right] \geq C i^{-n / 2} \exp \left(-\frac{c}{i}\left\|g_{\ell, i}^{\prime}-i m\right\|^{2}\right) .
$$

Modifying $c$ and $C$ if necessary, we obtain

$$
\inf _{x:\|x\| \leq r_{0} \text { and } g_{\ell, i}^{\prime} \in G} \mathbb{P}_{x}\left[S_{i}=g_{\ell, i}^{\prime}\right] \geq C \ell^{-n / 2} \exp \left(-c \ell^{2 \alpha-1}\right) .
$$

We apply this estimate to a path of the random walk starting at time $d \ell+k\left[(d \ell)^{\alpha}\right]$ from the point $x+\left(d \ell+k\left[(d \ell)^{\alpha}\right]\right) m$. Using (42) et (43) we obtain

$$
\begin{gathered}
\mathbb{P}\left[S_{1} \in \mathcal{C}, \ldots, S_{d \ell+k\left[(d \ell)^{\alpha}\right]} \in \mathcal{C},\left\|S_{d \ell+k\left[(d \ell)^{\alpha}\right]}-\left(d \ell+k\left[(d \ell)^{\alpha}\right]\right) m\right\| \leq r_{0},\right. \\
\left.S_{d \ell+k\left[(d \ell)^{\alpha}\right]+i}=g_{d \ell+k\left[(d \ell)^{\alpha}\right]+i}\right] \geq \frac{p}{2} \rho^{(d \ell)^{\alpha}} \times C \ell^{-n / 2} \exp \left(-c \ell^{2 \alpha-1}\right) .
\end{gathered}
$$

Moreover, we remember that, by the choice of $d$, if $\left\|S_{d \ell+k\left[(d \ell)^{\alpha}\right]}-\left(d \ell+k\left[(d \ell)^{\alpha}\right]\right) m\right\| \leq r_{0}$ then $S_{d \ell+k\left[(d \ell)^{\alpha}\right]+j} \in \mathcal{C}$ for all $j$ between 1 and $2 \ell$. We conclude that

$$
\mathbb{P}\left[S_{1} \in \mathcal{C}, \ldots, S_{d \ell+k\left[(d \ell)^{\alpha}\right]+i} \in \mathcal{C}, S_{d \ell+k\left[(d \ell)^{\alpha}\right]+i}=g_{d \ell+k\left[(d \ell)^{\alpha}\right]+i}\right] \geq \frac{p}{2} \rho^{(d \ell)^{\alpha}} C \ell^{-n / 2} \exp \left(-c \ell^{2 \alpha-1}\right) .
$$

Since $\alpha<1$, the $\left(d \ell+k\left[(d \ell)^{\alpha}\right]+i\right)^{-\alpha}$-th power of the right hand side has a positive limit when $\ell$ goes to infinity. This gives the announced conclusion, modulo the final easy following claim: all large enough integer can be written under the form $d \ell+k\left[(d \ell)^{\alpha}\right]+i$ with $0<\ell \leq i \leq 2 \ell$ 


\section{References}

[1] P. Biane, P. Bougerol and N. O'Connell, Littelmann paths and Brownian paths, Duke Math. J., 130 (2005), no. 1, 127-167.

[2] P. Billingsley, Convergence of Probability Measures, John Wiley and Sons (1968), second edition (1999).

[3] N. Bourbaki, Groupes et algèbres de Lie, Chapitres 4,5 et 6, Hermann (1968).

[4] H. Carlsson and S. Wainger, On the Multi-Dimensional Renewal Theorem, J. Math. Anal. Appl. 100 (1984), no. 1, 316-322.

[5] W. Fulton, Young tableaux, London Mathematical Society, Student Text 35.

[6] R. Garbit, Contributions à l'étude d'une marche aléatoire centrifuge et théorèmes limite pour des processus aléatoires conditionnés, Thèse de doctorat de l'Université François-Rabelais, Tours, 2008.

[7] R. Garbit, Temps de sortie d'un cône pour une marche aléatoire centrée, [Exit time of a centered random walk from a cone] C. R. Math. Acad. Sci. Paris 345 (2007), no. 10, 587-591.

[8] D. Grabiber and P. Magyar, Random walks in Weyl chambers and the decomposition of tensor products powers, J. Alg. Comb. 2 (1993), 239-260.

[9] I. A. Ibragimov And Yu. V. Linnik, Independent and stationary sequences of random variables, Translation from the Russian edited by J. F. C. Kingman. Wolters-Noordhoff Publishing, Groningen, 1971.

[10] B. Hall, Lie Groups, Lie Algebras, and Representations: An Elementary Introduction, Springer (2004).

[11] J. Hong, S. J. KAnG, Introduction to quantum groups and crystals bases, Graduate studies in Mathematics, 42 Amer. Math. Soc. (2002).

[12] S.J. Kang, K.C. Misra, Crystal bases and tensor product decompositions of $U_{q}\left(G_{2}\right)$ modules, Journal of Algebra, 163 (1994), 675-691.

[13] M. Kashiwara, On crystal bases, Canadian Mathematical Society, Conference Proceedings, 16 (1995), 155-197.

[14] M. Kashiwara, T. Nakashima, Crystal graphs for representations of the $q$-analogue of classical Lie algebras, J. Algebra, 165 (1994), 295-345.

[15] C. Lecouvey, Schensted-type correspondence, Plactic Monoid and Jeu de Taquin for type $C_{n}$, Journal of Algebra, 247 (2002), 295-331.

[16] C. Lecouvey, Schensted-type correspondences and plactic monoids for types $B_{n}$ and $D_{n}$, Journal of Algebraic Combinatorics, vol $18 \mathrm{n}^{\circ} 2$ (2003), 99-133.

[17] C. Lecouvey, Combinatorics of crystal graphs for the root systems of types $A_{n}, B_{n}, C_{n}, D_{n}$ and $G_{2}$, Combinatorial aspect of integrable systems, 11-41, MSJ Mem., 17, Math. Soc. Japan, Tokyo, 2007. 
[18] C. Lecouvey, E. Lesigne And M. Peigné, Le théorème de renouvellement multidimensionnel dans le "cas lattice". Preprint, hal-00522875.2010.

[19] N. O' Connell, A path-transformation for ramdom walks and the Robison-Schensted correspondence, Trans. Amer. Math. Soc., 355 (2003), 3669-3697.

[20] N. O' Connell, Conditioned random walks and the RSK correspondence, J. Phys. A, 36 (2003), 3049-3066.

[21] R. Proctor, Reflexion and an algorithm proofs of some Lie group dual pair identities, J. Comb. Th. A, 62 (1993), 107-127

[22] V. Rihter, Mehrdimensionale lokale Grenzwertsätze für grosse Abweichungen. (Russian. German summary) Teor. Veroyatnost. i Primenen 3 (1958) 107-114.

V. Richter, Local limit theorems for large deviations, Theor. Prob. Appl. 2 (1957) 206-219.

[23] R. Stanley, On the enumeration of skew Young tableaux, Advances in Applied Math., 30 (2003), 283-294.

[24] W. Woess, Denumerable Markov Chains, EMS Texbooks in Mathematics, (2009). 